Research Article

\title{
Characterization of Flow Interactions in a One-Stage Shrouded Axial Turbine
}

\author{
Adel Ghenaiet 1 \\ Laboratory of Energetic Mechanics and Conversion Systems, Faculty of Mechanical Engineering, University of Sciences and \\ Technology Houari Boumediene, BP32 El-Alia, Bab-Ezzouar, 16111 Algiers, Algeria \\ Correspondence should be addressed to Adel Ghenaiet; ag1964@yahoo.com
}

Received 12 May 2018; Revised 8 August 2018; Accepted 27 August 2018; Published 28 November 2018

Academic Editor: Giacomo V. Iungo

Copyright (c) 2018 Adel Ghenaiet. This is an open access article distributed under the Creative Commons Attribution License, which permits unrestricted use, distribution, and reproduction in any medium, provided the original work is properly cited.

\begin{abstract}
The aim of this paper is to characterize the steady and unsteady flow interactions through a one-stage high-pressure (hp) shrouded axial turbine with a tip cavity. The vane and blade passages were reduced based on the scaling technique, and the domains of compromise were identified and used in the flow computations. The flow structures are mainly in the form of vanes' wakes and vortices inducing circumferential distortions and interacting with the rotor blades. Fast Fourier transform (FFT) of the static pressure fluctuations recorded at the selected points and lines through the turbine stage revealed high unsteadiness characterized by a space-time periodic behavior, and described by the double Fourier decomposition. The vane-rotor interactions (VRI) appeared in the form of a potential flow field about the blades extending both upstream and downstream and correlated with the rotational speed. The other sources of unsteadiness are induced in the rotor blades by the vanes' wakes and referred to as the wake interaction, in addition to the secondary flows and vortices in endwall regions.
\end{abstract}

\section{Introduction}

High-pressure axial turbines are designed at high loading factors, leading to inherently complex flows which are in essence unsteady. The phenomena of vane/rotor interaction (VRI) arise from the displacement of the rotating blades against the stationary vanes, thereby complicating the mechanism of losses generation. One most important source of unsteadiness is the potential effect in which the pressure field associated with the leading edge of a blade sweeps past the trailing edge of a vane [1]. The other main contributor to unsteadiness is the vanes wakes swept into the blade row due to periodic chopping of wakes [2], added to the secondary flows and vortices convected from an upstream row [3]. Moreover, large variations in the size and strength of the secondary flows and vortices are observed as the rotor blades passages sweep through the flow distortions generated by upstream vanes [4]. Early experimental studies of wake/rotor interactions in axial turbines were performed by Hodson [2] and Binder et al. [5]. According to Sharma et al. [4, 6] and Arndt [7] the phenomena of VRI are presumably one of the difficult investigations to carry out both experimentally and numerically. Miller et al. [8] investigated the effect of the neighboring blade row on the pressure field around an hp rotor blade and showed, in addition to upstream and downstream interactions, a new interaction between downstream vane and upstream vane potential fields. There are only a few publications reporting the vortex-blade interaction mechanism for the shrouded axial turbines compared to unshrouded low aspect ratio axial turbines, such as the ones published by Chaluvadi et al. [9] and Schlienger et al. [10], which indicate that the unsteady secondary flows are primarily dominated by the rotor hub passage vortex and the secondary flows from the upstream vane. For the consideration of unsteady interactions between the leakage flows and the adjacent vanes/blade row, publications are rare; the only one noticed is that due to Qi and Zhou [11] who claimed that an upstream wake may reduce the strength of the tip leakage vortices.

Despite the possession of CFD codes providing acceptable flow details, the computation of unsteady flows in entire blade rows is still prohibitive. Therefore, there is a need for reducing the computational domain to save the 


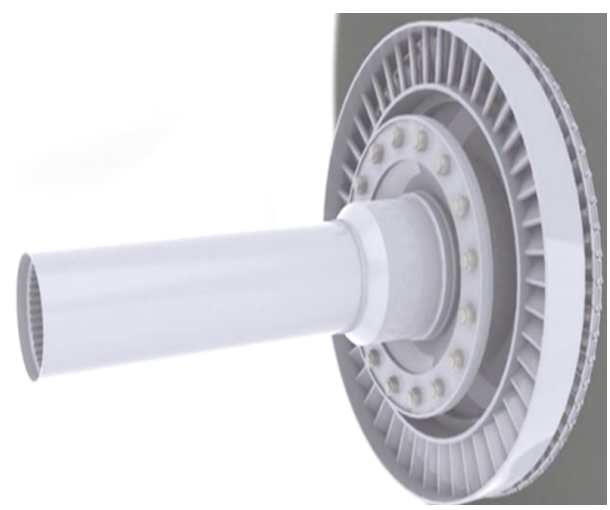

FIGURE 1: Hp shrouded axial turbine stage.

computing resources and time without affecting too much the flow physics. Periodic parts of a blade row may serve for such simplifications, but this is not usually possible owing to different vane/blade counts. Therefore, several developed techniques may account for the nonintegral blade counts without modeling the entire blade rows, such as the phase-lagged approach modified by Giles [12] to eliminate the assumption of temporal periodicity. To overcome the problem of nonintegral blade counts while maintaining a constant solidity, Rai and Madavan [13] developed the scaling technique. Arnone and Pacciani [14] based on the scaling method performed 2D unsteady flow analysis in the first stage of a transonic turbine consisting of 22 vanes and 38 blades and showed that the configuration $1: 2$ ( 1 vane and 2 blades) led to a premature choking of rotor blades passages and a lowering of exit vane velocity with an alteration of pressure distribution, which were nonexistent in the configuration 4:7. Also, Clark et al. [15] completed 3-D unsteady simulations in a 1.5-stage hp axial turbine of vane/ blade counts 36,56 , and 36 , through a quarter of an entire domain and a simplified domain $1: 18$ consdiering the scaling technique. As a result, the time-averaged pressure distributions were not affected, contrary to the pressure fluctuations. Furthermore, Yao et al. [16] simulated 3-D unsteady flows in a 1.5stage axial turbine of blade counts 36,41 , and 36 and arrived to the same conclusions.

The aim of this paper is to investigate the aerodynamic performance and the VRI phenomena through an hp shrouded axial turbine with a tip cavity. The scaling technique allowed creating several reduced multichannel domains. Following the analyses of expansion properties and flow structures as well as the computing cost, the optimal computational domain was identified and adopted. After studying the steady aerodynamic performance and flow structures, the unsteady flow simulations served to characterize the fluctuations of static pressure, both in time and space, at different monitoring points and lines through the components. By means of FFT analysis, the different frequencies and spatial modes of components interactions and their origins were revealed.

\section{CFD Model}

The single-stage hp axial turbine (Figure 1) is a component from a mixed turbofan consiting of an NGV of 46 vanes and a rotor with 64 shrouded blades with a tip cavity. The main geometry data are summarized in Table 1. The reproduced CAD models of NGV and rotor are shown by Figure 2. At the takeoff operating conditions, the engine parameters correspond to a rotational speed of 12,250 rpm, total air mass flow of $146 \mathrm{~kg} / \mathrm{s}$, bypass ratio of 1.03 , pressure ratio of 15.5 , and turbine inlet temperature of $1335.15 \mathrm{~K}$ [17].

2.1. Solver and Setting. ANSYS CFX-solver [18] is used to solve URANS equations (as below) in the finite volume environment. The computational domain has an upstream duct connecting the combustion chamber to NGV and a shrouded rotor blade with a tip cavity (Figure 2):

$$
\begin{aligned}
\frac{\partial \rho}{\partial t}+\frac{\partial}{\partial x_{j}}\left(\rho U_{j}\right)=0 & \\
\frac{\partial}{\partial t}\left(\rho U_{i}\right)+\frac{\partial}{\partial x_{j}}\left(\rho U_{j} U_{i}\right)= & -\frac{\partial P}{\partial x_{i}}+\frac{\partial}{\partial x_{j}} \\
& \cdot\left[\tau_{\mathrm{ij}}-\rho \overline{u_{\iota} u_{J}}\right]+S_{i} \\
\frac{\partial}{\partial t}\left(\rho h_{t}\right)-\frac{\partial P}{\partial t}+\frac{\partial}{\partial x_{j}}\left(\rho U_{j} h_{t}\right)= & \frac{\partial}{\partial x_{j}}\left(\lambda \frac{\partial T}{\partial x_{j}}-\rho \overline{u_{j} h}\right) \\
& +\frac{\partial}{\partial x_{j}}\left[U_{i}\left(\tau_{i j}-\rho \overline{u_{i} u_{j}}\right)\right] \\
& +S_{E},
\end{aligned}
$$

where the stress tensor $\tau=\mu\left(\nabla U+(\nabla U)^{t}-(2 / 3) \delta \nabla U\right)$, $\vec{S}=-\rho(2 \vec{\Omega} \times \vec{U}+\vec{\Omega} \times(\vec{\Omega} \times \vec{r}))$ : Coriolis and centrifugal force terms. $h_{t}=h+U_{i} U_{i} / 2+k$ total enthalpy and $k=(1 / 2)$ $\overline{u_{i}^{2}}, k$ turbulence kinetic energy, $S_{E}$ energy source term. These equations are integrated over each control volume defined by joining the edge centres and the element centres surrounding each node. The volume integral is evaluated by considering the flow properties as constant and equal to the central value (mesh node), whereas the surface integral is evaluated at the integration points located at the centres of surface segments.

The boundary conditions, summarized in Table 2 , include the total pressure and temperature (takeoff operating conditions [17]) imposed at the inlet of NGV, while downstream of the rotor blade, a static pressure was varied to sweep the totality of the mass flow rate for different rotational speeds. Moreover, a free stream turbulence intensity $\mathrm{Tu}=(2 k / 3)^{1 / 2} /$ $V$ was imposed at the inlet, where $V$ is a representative velocity and $k$ denotes the turbulence kinetic energy of incoming flow which the intensity depends on the inlet vane length scale. The turbulent viscosity is evaluated from Wilcox's model [19].

The steady flow solution (RANS equations) used the high resolution scheme. To prevent pressure field oscillations due to a nonstaggered collocated grid, a coupled solver solves the flow equations as a single system using the SIMPLE algorithm in conjunction with the interpolation technique for the momentum. The solution method is fully implicit, and the 
TABLE 1: Geometry parameters.

\begin{tabular}{lccc}
\hline Parameters & & NGV & Rotor \\
\hline Vane/blade count & & 46 & 64 \\
Inlet minimal diameter & $(\mathrm{mm})$ & 490.5 & 487.5 \\
Inlet maximal diameter & $(\mathrm{mm})$ & 644 & 647.5 \\
Outlet minimal diameter & $(\mathrm{mm})$ & 487.5 & 476 \\
Outlet maximal diameter & $(\mathrm{mm})$ & 647.5 & - \\
Tip clearance & $(\mathrm{mm})$ & $48.5-50.6$ & $656.3 / 665.5$ (with cavity) \\
Axial chord (min, max) & $(\mathrm{mm})$ & 40 & 1.45 \\
Twist angle at hub at shroud & $(\mathrm{deg})$ & 37.5 & 24 \\
Camber angle & $(\mathrm{deg})$ & 67 & 40 \\
\end{tabular}

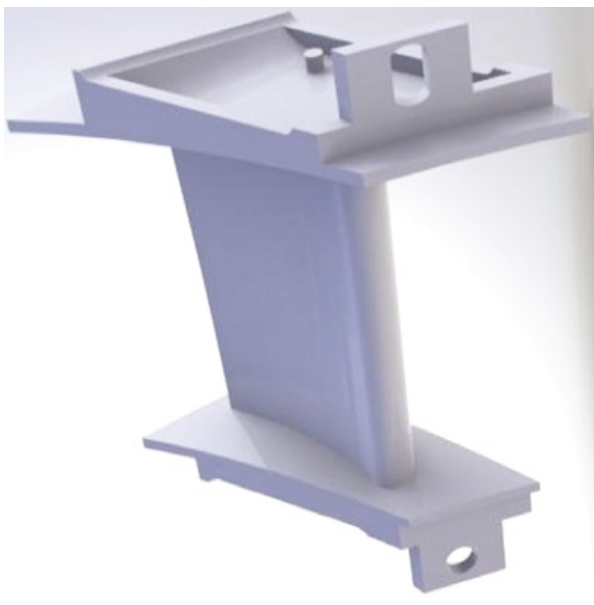

(a)
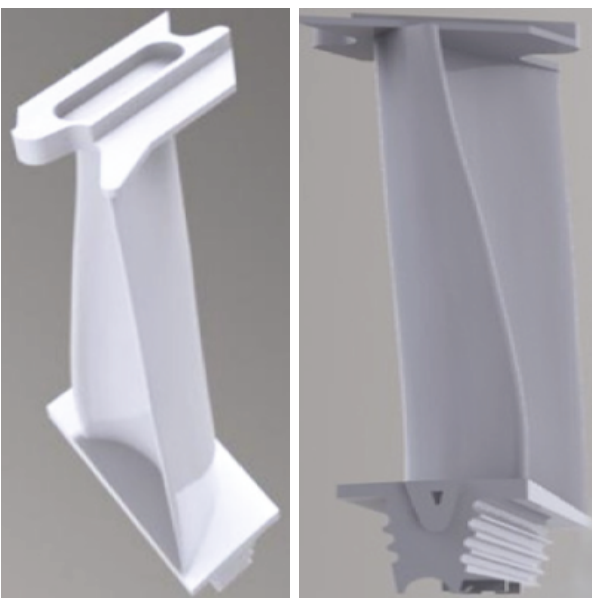

(b)

Figure 2: CAD view of (a) NGV and (b) rotor blade with tip cavity.

TABLE 2: Boundary conditions.

\begin{tabular}{|c|c|c|c|c|}
\hline Fluid & \multicolumn{4}{|c|}{ Combustion gas mixture } \\
\hline Inlet conditions & \multicolumn{4}{|c|}{$\begin{array}{l}\text { Total pressure } 1.539 \mathrm{MPa} \& \text { total temperature }=1335.15 \mathrm{~K} \\
\text { Turbulence intensity } 5 \% \text { \& length scale }=0.1 \times h\end{array}$} \\
\hline Outlet conditions & \multicolumn{4}{|c|}{ Static pressure: $\mathrm{Pa}$} \\
\hline \multirow{4}{*}{ Wall conditions } & & NGV & \multicolumn{2}{|r|}{ Rotor } \\
\hline & Vane & No slip stationary wall & Blade & No slip rotating wall \\
\hline & Hub & No slip stationary wall & Hub & No slip stationary wall \\
\hline & Shroud & No slip stationary wall & Shroud & Counterrotating wall \\
\hline Lateral surfaces & \multicolumn{4}{|c|}{ Periodicity } \\
\hline Interface in steady simulations & \multicolumn{4}{|c|}{ Frozen and stage interface } \\
\hline Interface in unsteady simulations & \multicolumn{4}{|c|}{ Transient rotor/stator interface } \\
\hline
\end{tabular}

time step acts as a relaxation parameter for a convergence residual inferior to $10^{-6}$.

Multiple reference frames differ in the way the interface between moving and nonmoving cell zones is treated. In the frozen rotor model, the coupling between the cell zones is done by just switching the absolute velocity between relative and absolute frames, and thus, a snapshot of the flow field is obtained with the consideration of wakes between different cell zones. Presently, the frozen rotor interface was used to characterize the flow details of the turbine stage. In the mixing plane model (stage interface), the steady state solution is calculated for each fluid zone, and the two 
TABLE 3: Fraction of laminar flow of different components.

\begin{tabular}{lcccccccc}
\hline & $C(\mathrm{~mm})$ & $V$ or $W(\mathrm{~m} / \mathrm{s})$ & $\rho\left(\mathrm{kg} / \mathrm{m}^{3}\right)$ & $\mu(\mathrm{Pa} \cdot \mathrm{s}) \times 10^{-5}$ & $K(\mathrm{~J} / \mathrm{kg})$ & $\mathrm{Tu}(\%)$ & $\operatorname{Re}_{c} \times 10^{6}$ & Fraction of laminar flow $(\%)$ \\
\hline NGV & 49.55 & 525 & 3.128 & 1.83 & 350 & 2.91 & 4.446 & 2.936 \\
Rotor & 34.05 & 395 & 2.613 & - & 750 & 5.66 & 1.920 & 3.495 \\
\hline
\end{tabular}

adjacent frames are coupled by an exchange of the flow field variables at the interface. The flow field data are averaged circumferentially for both frames at the interface and passed to the adjacent zone as boundary conditions. This spatially averaging technique at the interface removes any unsteadiness that would arise due to variations in the zone-to-zone flow field. Herein, the mixing plane model is used to determine the steady aerothermodynamic performance. The transient rotor/stator interface model accounts for the frame of reference transfer and pitch changes, and assumes that the flow field is unsteady, thus can model the interactions between vanes and blades. The relative motion is simulated through the implemented general grid interface (GGI) connection permitting the nonmatching of node locations, element type, and surface overlap among others.

Unsteady flow solution (URANS equations) used the second order backward Euler as a transient scheme with a high-resolution advection scheme. The transient simulations consist in physically advancing the flow in a real time, and because it was not possible to use the final time step's flow field alone to assess the convergence in terms of RMS/MAX residuals and imbalances, some sort of average was required over an appropriate timescale. The number of iterations per time step corresponed to 10 iterations for one inner loop to achieve the accuracy of the residual (RMS $\leq 10^{-6}$ ) using the selected time step which is small enough to get the necessary time resolution of unsteady VRI depending on the speed of rotation. The different characteristic timescales are estimated as follows:

(i) Necessary time to accomplish one rotor round is $\Delta t_{\text {round }}=60 / n$

(ii) Apparent blade passing period is $\Delta t_{i}=\Delta t_{\text {round }} / N_{3-i}$, $i=1,2$, where $N_{3-i}$ is the blade number of row $(3-i)$

(iii) Necessary time to cover geometrical coincidence is given by $\Delta t_{\text {min }}=\Delta t_{\text {round }} \operatorname{GCD}\left(N_{i}, N_{3-i}\right) / N_{i} N_{3-i}$, where $\operatorname{GCD}\left(N_{i}, N_{3-i}\right)$ is the greatest common divisor

For this hp single-stage axial turbine consisting of 46 vanes and 64 blades and operating at a nominal speed of rotation of $12,242 \mathrm{rpm}, \Delta t_{\text {round }}=4.901 \mathrm{~ms}$ and thus $\Delta t_{\min }=$ $3.3295 \mu$ s equivalent to 0.2445 degrees.

2.2. Turbulence Model. The conventional turbulence models offer a good balance between grid resolution requirement and flow field complexity; on the other hand, the large eddy simulation is still prohibitive and probably too detailed to be used for engineering design purposes [20]. Zhou et al. [21] used several turbulence models such as Spalart-Allmaras, standard $\mathrm{k}-\varepsilon$, $\mathrm{k} \omega$-SST, and transition $\mathrm{k} \omega$-SST in studying the effect of the trailing edge of ultrahigh lift lp turbine blades and showed that the two last models provided a good prediction of aerodynamic performance compared with experiments. Moreover, the $\mathrm{k} \omega$-SST model has proven certain reliability in studying the flow interactions with a good revelation of pressure oscillations [22]. The transition modeling represents a critical matter for industrial applications; however, according to the literature, this requires the solution of two extra equations with an additional CPU time about $18 \%$, as well as fine grids with a maximum value $y^{+}$ $\leq 1$, to resolve the laminar boundary layers and sufficient grid points in the streamwise direction to resolve the transitional region. Herein, the dimensionless parameter $y^{+}=$ $(\Delta y / v) U_{t}$ considers the friction law of a flat plate $U_{t}=$ $V_{\infty} \sqrt{C_{f} / 2}$ and $C_{f}=0.037 \mathrm{Re}_{c}^{-1 / 5}$ with $\operatorname{Re}_{c}=\rho V_{\infty} c / \mu$, where $c$ is the chord. In general, the fraction (percentage) of laminar flow can be estimated from $\mathrm{Re}_{x t} / \mathrm{Re}_{c}=380,000$ $(100 \mathrm{Tu})^{-5 / 4} / \operatorname{Re}_{c}$, based on the empirical correlation for the transition onset (Mayle [23]), where $\operatorname{Re}_{x t}$ is the transition Reynolds number. In an hp axial turbine, the fraction of laminar flow is small, and since the aerodynamic losses are mostly attributed to the turbulent flow after transition, the effect of transition on losses is not significant [23]. The estimations of the fractions of laminar flow are based on the mid vane/blade chord and flow properties averaged at the exit. Table 3 shows small fractions of laminar flow, because of high flow velocities, and as consequence the adopted $k \omega$-SST model was selected, which allows switching between the standard wall function (for $20<y^{+}<100$ ) and the low Reynolds number model (for $y^{+}<2$ ) to resolve the details of boundary layers.

2.3. Mesh Generation. Unstructured blocks of H-grids and O-grids (Figure 3 ) were used, with refinements around the vane and blade, whereas the tip cavity (Figure 4) was meshed separately. The parameter $y^{+}$was used to check the distance of the first node from the vane or blade surfaces. To cover regions of high Reynolds number, the exit flow velocities from the vane or rotor blade correspond to the takeoff operating conditions.

The aerodynamic performances were assessed for seven grids of different sizes. The expansion performances show stabilization (Figure 5) above the sixth grid size, and hence, the overall grid size per one channel of the vane/blade is $667,088: 261,018$ nodes for one vane passage, and 250,486 nodes for one blade passage added the tip cavity of 155,584 nodes.

The consideration of the entire channels is very expansive and limited by the resolution of the grids, and this is why the computational domain was reduced by the scaling method. As shown by Table 4, four configurations of computational 


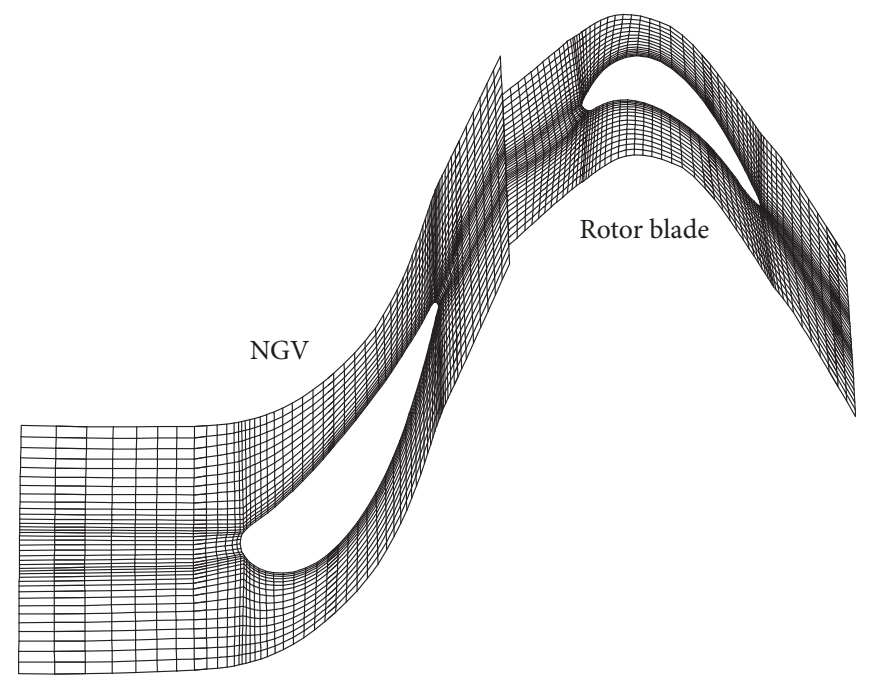

Figure 3: Midspan grids per one vane and one blade passages.

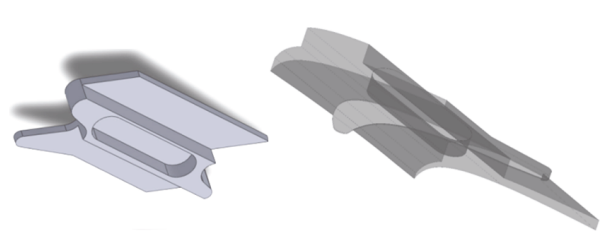

(a)

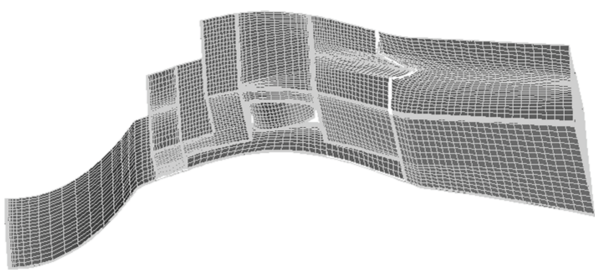

(b)

FIGURE 4: Tip clearance cavity: (a) geometry and (b) grid.

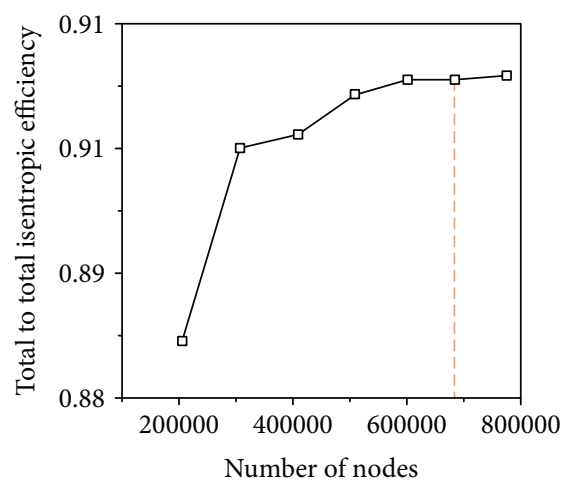

(a)

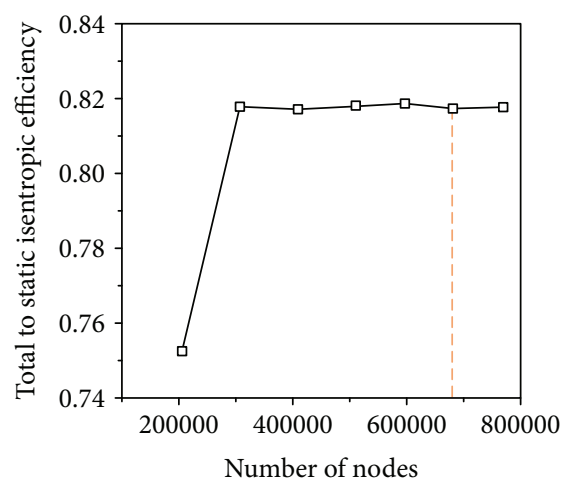

(b)

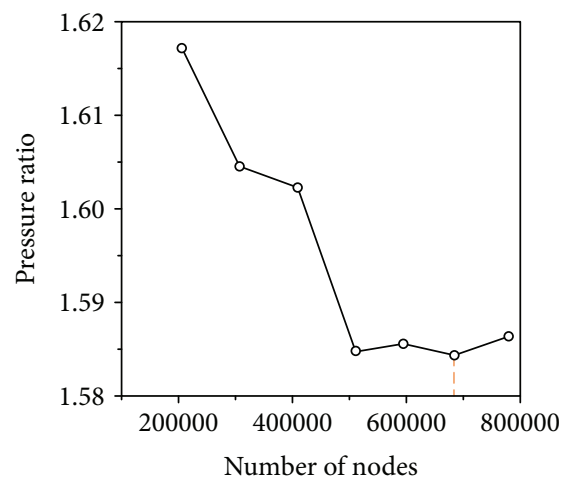

(c)

FIgURE 5: Grid dependency analysis: (a) total-to-total isentropic efficiency, (b) total-to-static isentropic efficiency, and (c) pressure ratio.

domains (Figure 6) were evaluated including: the baseline configuration consisting of a half of blade rows of 23 vanes and 32 blades, the configuration "NGV 11:16" consisting of 11 vanes and 16 blades (vane chord reduced by $4.5 \%$ ), the configuration "NGV $3: 4$ " consisting of 3 vanes and 4 blades (vane chord reduced by $4.2 \%$ ), and the last configuration "rotor $2: 3$ " consisting of 2 vanes and 3 blades (blade chord reduced by $7.25 \%$ ). The different grid sizes are listed in Table 4 depicting a scaling factor near unity which in turn affects slightly the blade pressure distribution and avoids an excessive grid size which would require longer simulation time.

2.4. Comparison between Reduced Domains. The three scalereduced domains are compared with the baseline in terms of entropy (obtained from the frozen rotor simulations) as depicted in Figures 7 and 8, and expansion properties and performance (based on the stage interface simulations) as depicted in Figures 9 and 10. The plots of static entropy in blade-to-blade from hub to tip at spans $10 \%, 50 \%$, and $90 \%$ 
TABLE 4: Scaled computational domains.

\begin{tabular}{|c|c|c|c|c|c|c|c|}
\hline & $\begin{array}{c}\text { Total vane } \\
\text { number }\end{array}$ & $\begin{array}{c}\text { Total blade } \\
\text { number }\end{array}$ & $\begin{array}{c}\text { Domain vane } \\
\text { number }\end{array}$ & $\begin{array}{c}\text { Domain blade } \\
\text { number }\end{array}$ & $\begin{array}{c}\text { Scaling } \\
\text { ratio }\end{array}$ & $\begin{array}{c}\text { Chord } \\
\text { variation }\end{array}$ & Grid size $\times 10^{6}$ \\
\hline Baseline & 46 & 64 & 23 & 32 & - & - & 19.5 \\
\hline NGV $3: 4$ & 48 & 64 & 3 & 4 & 0.958 & $-4.2 \%$ & 2.4 \\
\hline $\begin{array}{l}\text { NGV } \\
11: 16\end{array}$ & 44 & 64 & 11 & 16 & 1.045 & $4.5 \%$ & 9.3 \\
\hline Rotor $2: 3$ & 46 & 69 & 2 & 3 & 0.9275 & $-7.25 \%$ & 1.7 \\
\hline
\end{tabular}

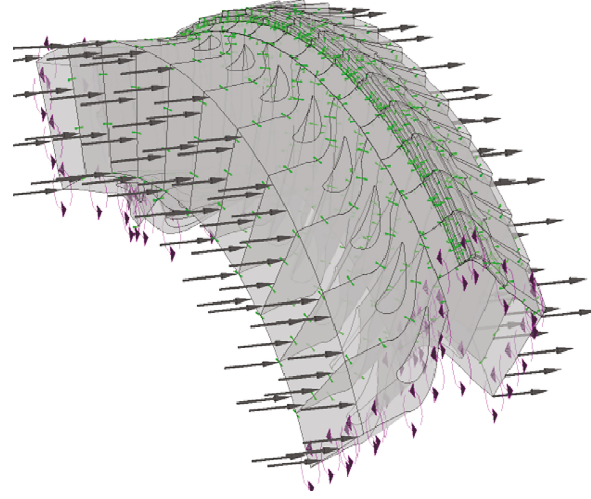

(a)

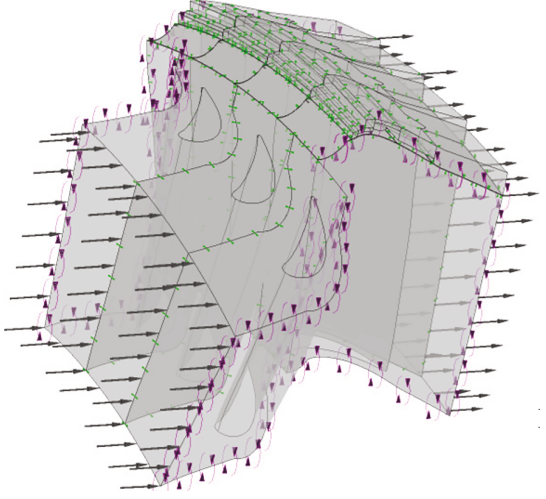

(b)

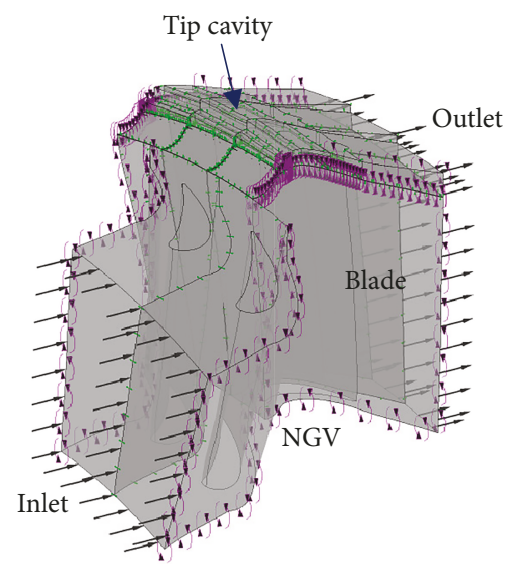

(c)

FIGURE 6: Scaled computational domains: (a) NGV $11: 16$, (b) NGV $3: 4$, and (c) rotor $2: 3$.

and in the tip cavity at spans $96 \%$ and $98.5 \%$ are shown by Figure 7 . It is evident that the vanes wakes convected inside the rotor blades differ by comparing between the baseline and the other reduced domains. The baseline (Figure $7(\mathrm{a})$ ) produced better resolution of flow details whereas the configuration rotor 2:3 produced Mach number over the suction side bit higher, especially at $50 \%$ and $90 \%$ of the span, explaining the difference in aerodynamic losses and loading. As the flow structure in the tip clearance and tip cavity of the configuration rotor 2:3 differs drastically, this in turn translates into a higher entropy generation at spans $96 \%$ and $98.5 \%$.

Figure 9 depicts streamwise variation of the averaged expansion properties. The configurations adopting scaling factors close to unity i.e. 0.958 for NGV 3:4 and 1.045 for NGV 11:16 seem not affecting too much the expansion properties. At the nominal point, there is almost the same trend compared with the baseline, confirming the good choice of the current scaling factors. However, with the configuration rotor $2: 3$, the expansion properties differ beyond $70 \%$ of the blade chord, showing that the scaling of the rotor blade induces more losses since there are more blades and tip cavities in addition to the modification of blade throat and its critical condition.

Furthermore, to show the circumferential flow perturbations downstream of vanes and blades, the contours of entropy are plotted in Figure 8. At the exit of vanes, the radial equilibrium of flow translates into a positive 

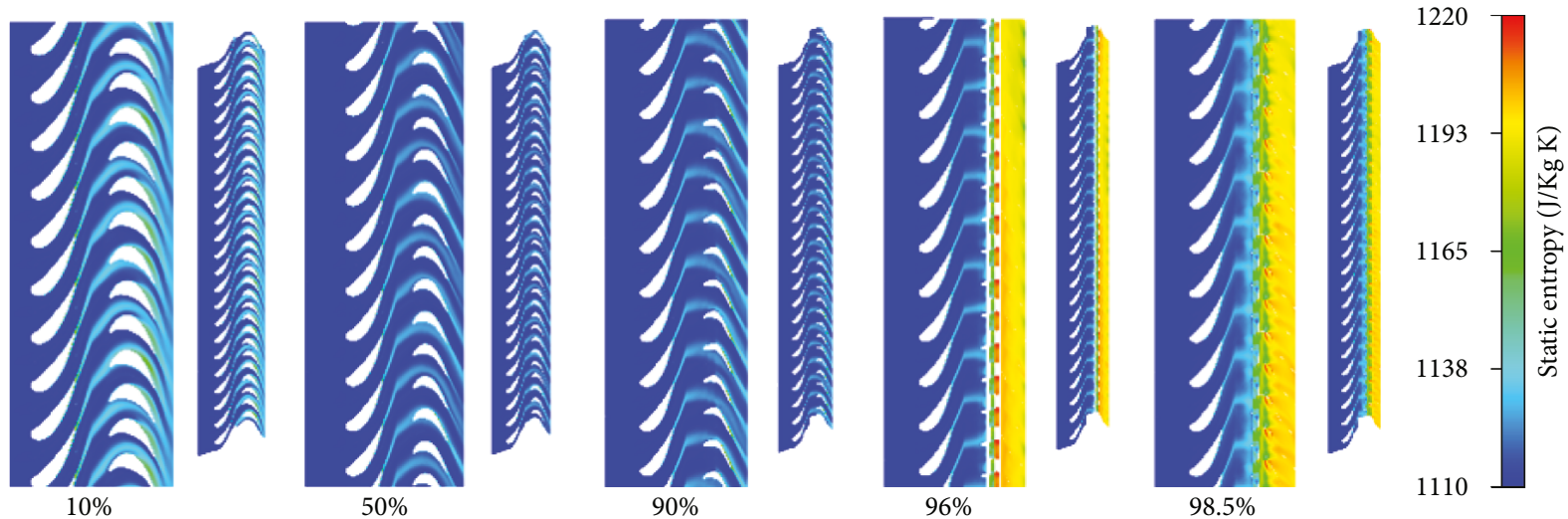

(a)
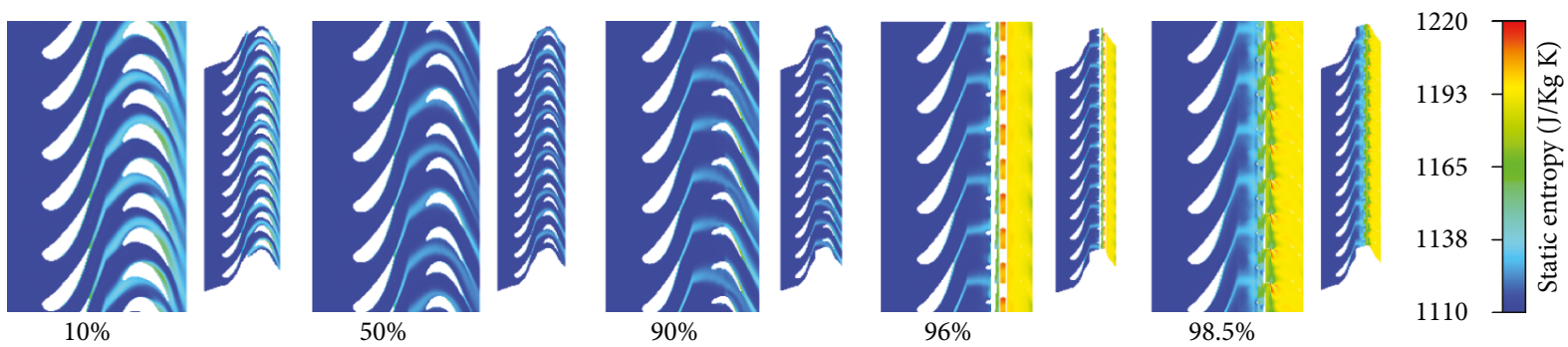

(b)
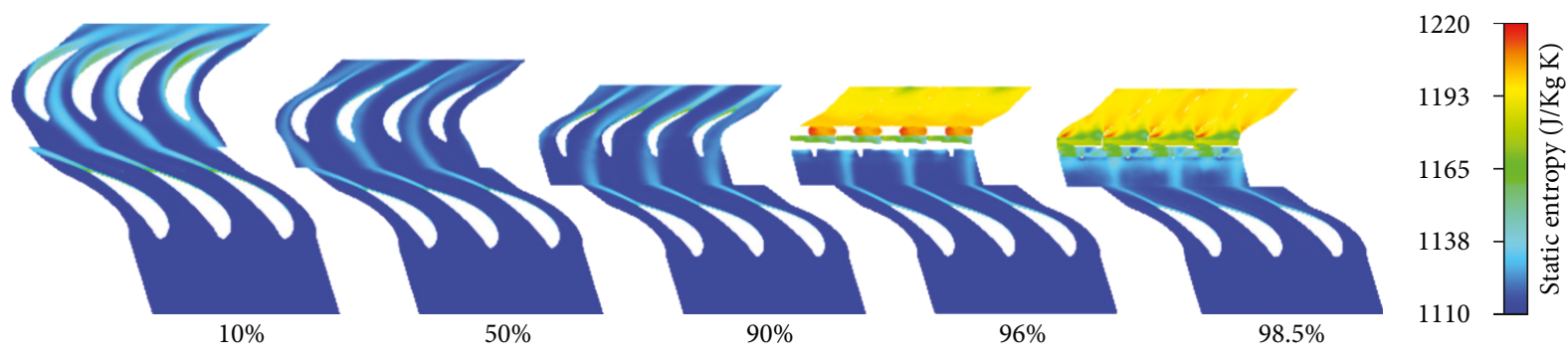

(c)

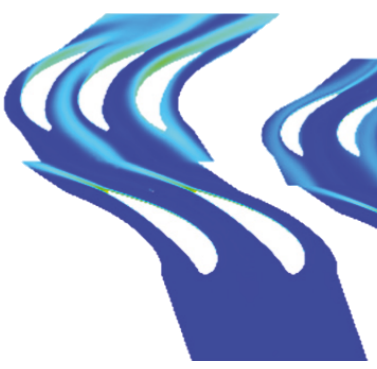

$10 \%$

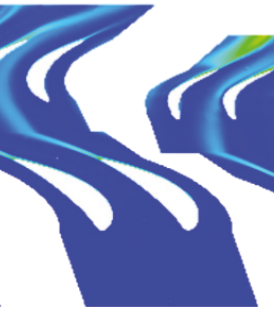

$50 \%$

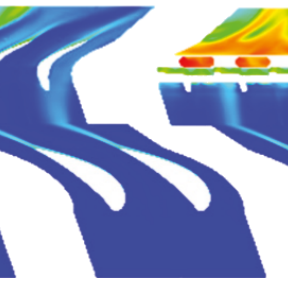

$90 \%$

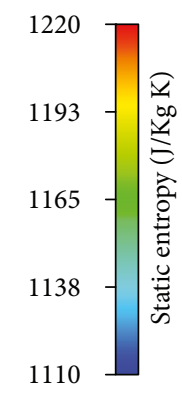

Figure 7: Entropy contours (at nominal point) at spans 10\%, 50\%, 90\%, 96\%, and 98\% (from left to right), successively: (a) baseline, (b) NGV $11: 16$, (c) NGV $3: 4$, and (d) rotor $2: 3$.

gradient of pressure from hub to shroud, and a radial distribution of entropy. Downstream of the rotor blades and due to different aerodynamic loadings, the radial equilibrium is affected differently and hence the flow structures. Moreover, the incoming vanes wakes and vortices are swept differently through the blade row, and this is why the structure of secondary flows and vortices near the hub and shroud corners differ completely.

The turbine stage performance computed for 100\% rotational speed shows that the expansion ratio (Figure 10(a)) for all the configurations are aligned with the baseline, but at the high mass flow rate the choke is reached earlier for rotor 


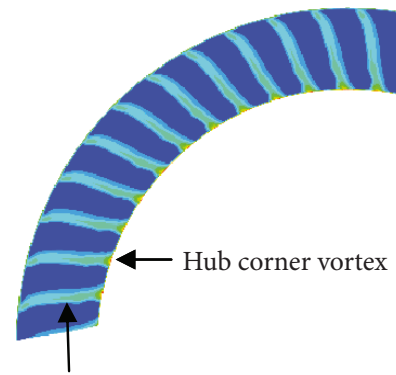

Vane wake

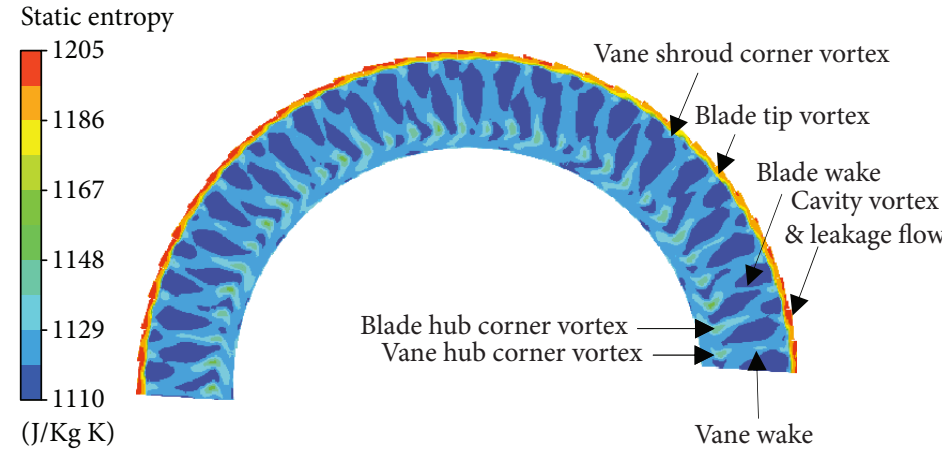

(a)
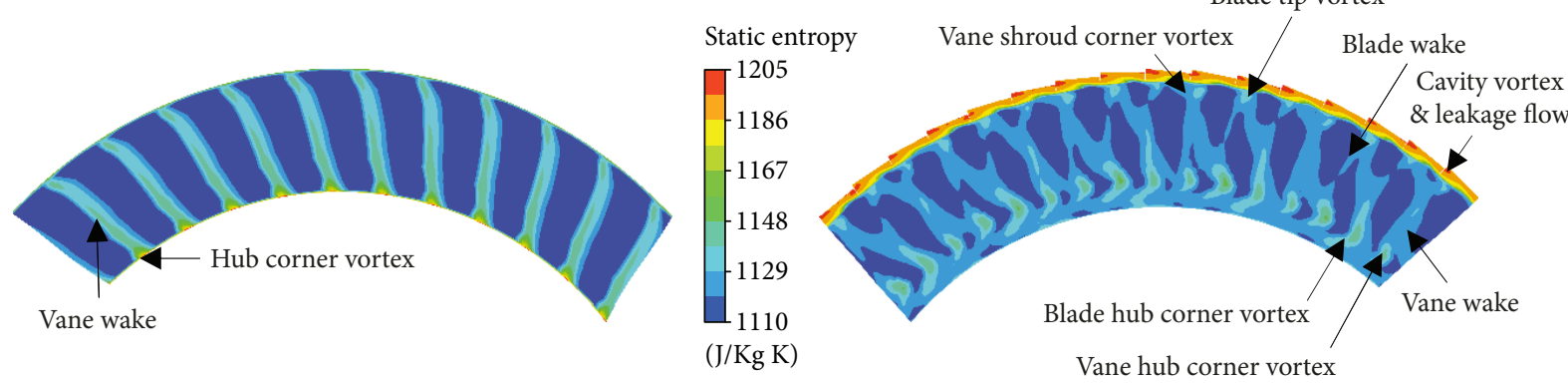

Blade tip vortex

(b)

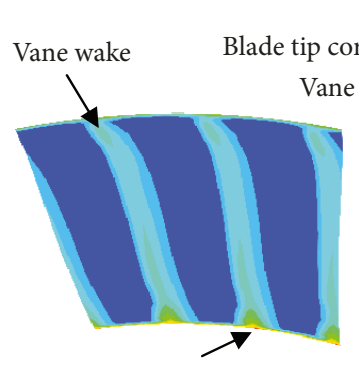

Hub corner vortex

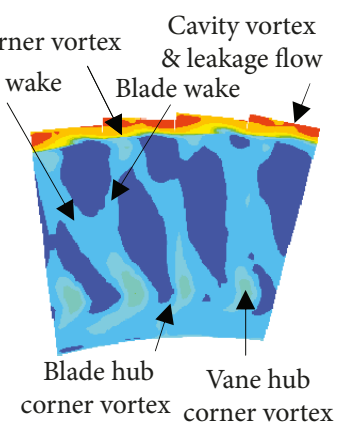

(c)

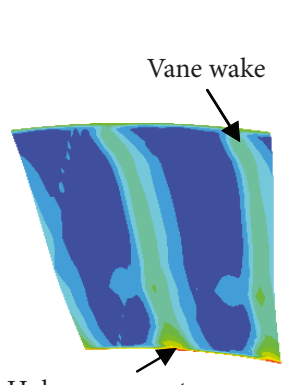

Hub corner vortex

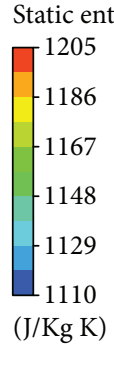

FIGURE 8: Entropy contours at exit planes of NGV and rotor blade (at nominal point) for (a) baseline, (b) NGV 11:16, (c) NGV 3:4, and (d) rotor $2: 3$.

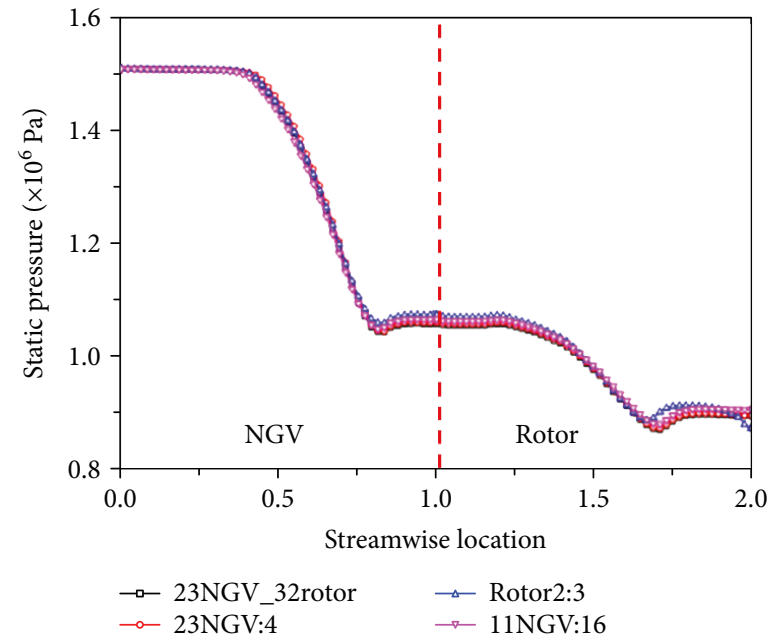

(a)

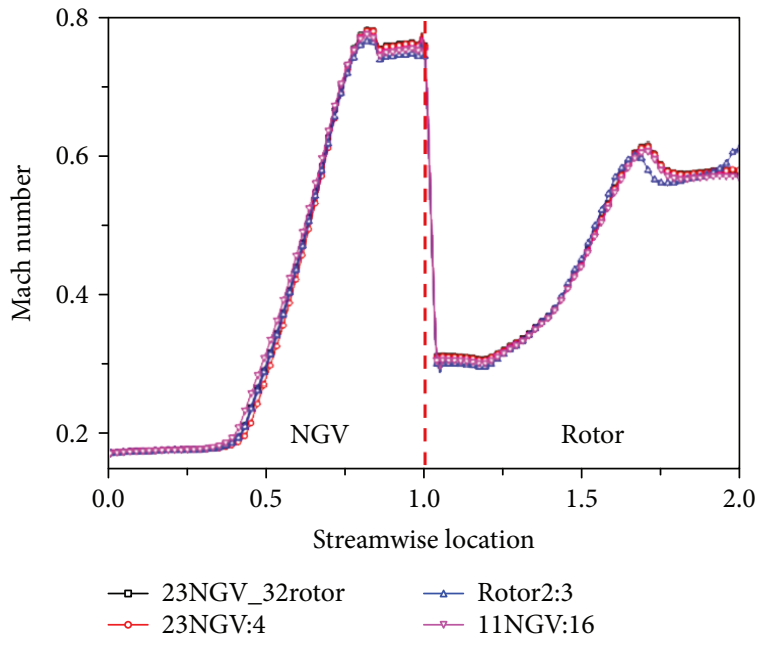

(b)

Figure 9: Streamwise variation of (a) static pressure and (b) relative Mach number. 


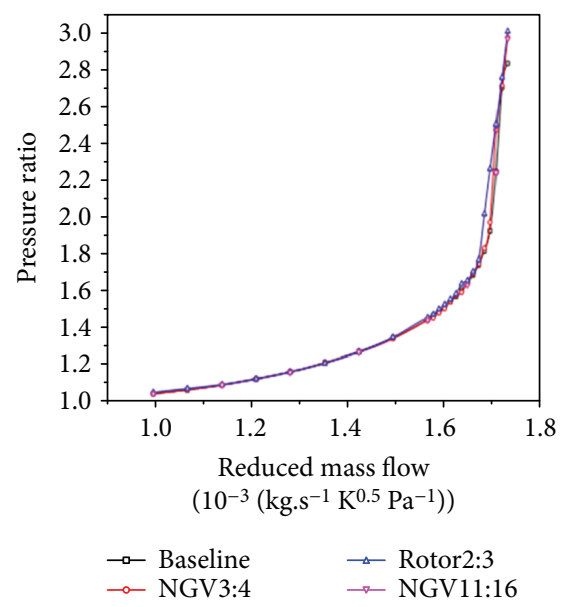

(a)

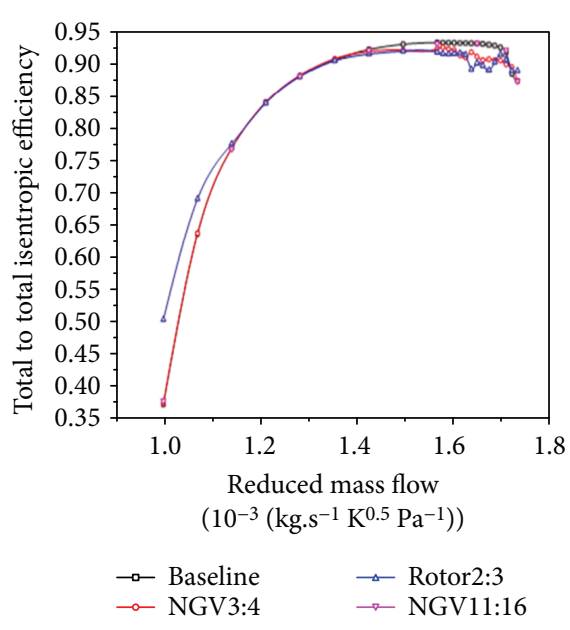

(b)

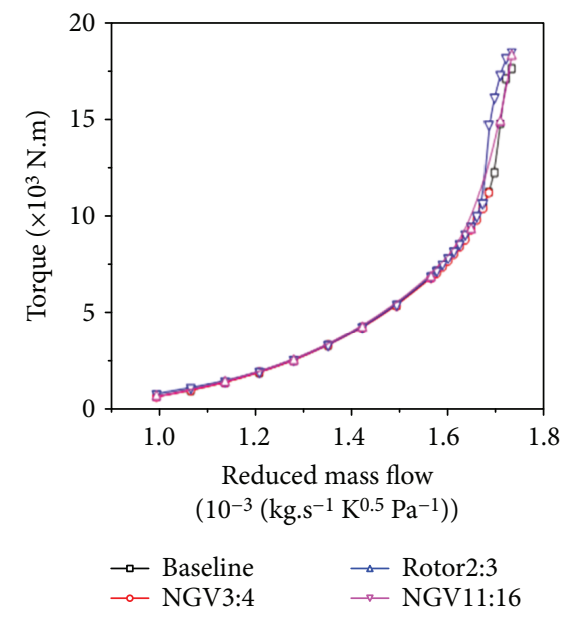

(c)

FIGURE 10: Turbine stage performance: (a) total-to-total pressure ratio, (b) total-to-total isentropic efficiency, and (c) torque.

$2: 3$ due to reduction in the throat area of the blade. The totalto-total isentropic efficiency curves (Figure 10(b)) follow practically the same trend at low mass flow rates but deviate slightly at high mass flow rates except for the configuration rotor 2:3 which the efficiency undulates at near choking, since the scaling affected the losses. The torque of the configuration rotor $2: 3$ differs (Figure $10(\mathrm{c})$ ) at high mass flow rates since there is no kinematic similarity maintaining the same speed of sound in the scaled configurations, and hence, the pressure distributions and aerodynamic loading are affected. Globally, it seems that there is a good agreement between NGV 11:16, NGV 3:4, and the baseline.

2.5. Computational Gain. The approximated number of nodes of the baseline configuration ( 23 vanes and 32 blades) is $19.5 \times 10^{6}$, for the upscaled (4.5\%) NGV $11: 16$ configuration is $9.3 \times 10^{6}$, for the downscaled (4.2\%) NGV $3: 4$ configuration is $2.4 \times 10^{6}$, and for the downscaled $(7.25 \%)$ configuration rotor $2: 3$ is $1.7 \times 10^{6}$. The preliminary unsteady flow simulations at the nominal operating point permitted evaluating the gain in the computing power. For the configurations of baseline and upscaled NGV 11:16, the average computing time was $14 \mathrm{~min}$ and $9 \mathrm{~min}$, respectively, per time step so that the total time for each simulation could reach 2184 days and 1404 days, respectively. Because of the cluster capacity did not allow writing down the data files, it was decided to limit the unsteady simulations to the configuration NGV $3: 4$ of a total nodes' number of 2,407,334 which constituted a good compromise. In this instance, the computations used a workstation of 7 processors and $16 \mathrm{~GB}$ of RAM, and each time step took about $3 \mathrm{~min}$ so that the total simulation time was 20 days.

\section{Steady Flow Results}

The steady performance maps are obtained based on the computational domain NGV $3: 4$ and by adopting the stage interface as the mixing plane. As depicted in Figure 11 for each rotational speed, the total-to-total expansion ratio $\left(\pi_{\mathrm{tt}}\right)$ initially varied moderately, increases rapidly to reach the choke limit which shifts with the speed of rotation. The limit of the expansion ratio reaches a maximum of 2.97 at the rotational speed $100 \%$ and a reduced mass flow rate of $1.73 \times 10^{-3}\left[\mathrm{~kg} \cdot \mathrm{s}^{-1} \mathrm{~K}^{0.5} \mathrm{~Pa}^{-1}\right]$ equivalent to a mass flow rate of $73 \mathrm{~kg} / \mathrm{s}$. The curves of efficiency $\left(\eta_{\mathrm{ttis}}\right)$ reveal a maximum of efficiency that increases slightly with the rotational speed. The nominal point corresponds to a maximum of $93.05 \%$ of total-to-total isentropic efficiency and $85.63 \%$ of total-to-static isentropic efficiency at the nominal speed and reduced mass flow rate of $1.638 \times 10^{-3}\left[\mathrm{~kg} \cdot \mathrm{s}^{-1} \mathrm{~K}^{0.5} \mathrm{~Pa}^{-1}\right]$ which corresponds to a mass flow of $69.5 \mathrm{~kg} / \mathrm{s}$. For this high aspect ratio hp turbine, the operating range is broad even at high rotational speeds.

3.1. Flow Structures. To characterize the flow structures at the nominal operating point, the configuration NGV $3: 4$ and the frozen rotor interface were used for the steady flow computations. The expansion through NGV and rotor occurs with a significant drop in static pressure and the flow accelerates beyond the throat in the form of expansion waves. After the throat and near the vane hub, an eddy is formed owing to interaction between the boundary layer and the expansion waves. At the rotor leading edge, the flow incidence increases from hub to shroud due to high peripheral speed and blade twist; hence, the stagnation point moves towards the blade pressure side near the hub and towards the suction side near the shroud to cause flow acceleration around the leading edge. Over the blade suction side from the hub and under the tip cavity, flow deviations are seen which, lead to the formation of secondary flows interacting with the main flow. As also noticed, the hub passage vortex mixes with the core flow up to $10 \%$ span. Inside the tip cavity, the flow is very slow with the formation of a trapped vortex, in addition to another region of decelerated flow due to mixing between leakage flow and free step vortex behind the tip cavity. At the $90 \%$ 


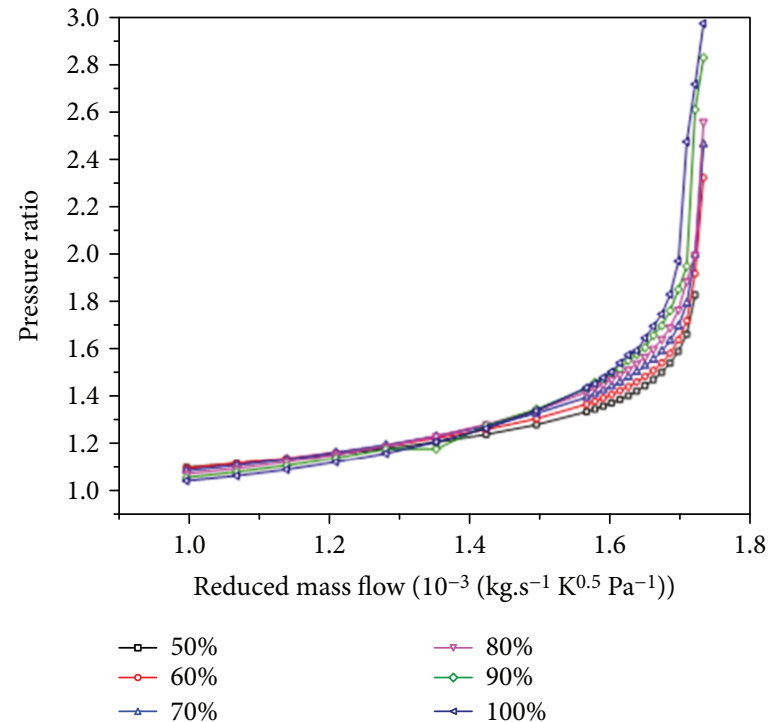

(a)

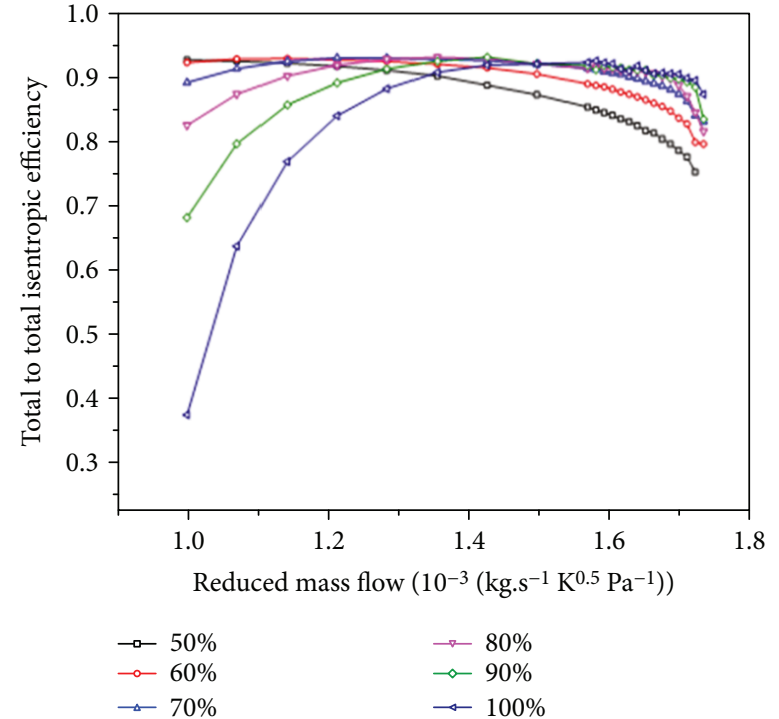

(b)

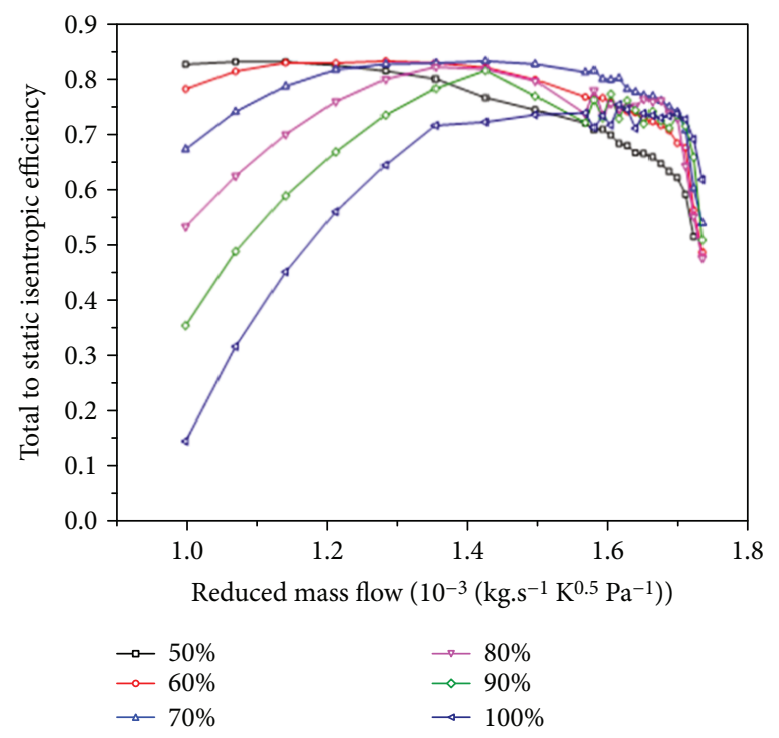

(c)

FIGURE 11: Turbine stage performance maps: (a) total-to-total pressure ratio, (b) total-to-total isentropic efficiency, and (c) total-to-static isentropic efficiency.

span, there is a jet-pointing band located on the suction side transmitted by the under cavity vortex but vanishes due to mixing with the main flow. Underneath the tip cavity, the negative incidence creates a slight flow separation on the pressure side just after the leading edge, and its interaction with the under cavity boundary layer leads to a vortex creation. The effect of tip cavity is clearly illustrated above the $96 \%$ span where the uniformly low static pressure is related to the leakage flow reduction. The highest value of static entropy is marked in the tip cavity as characterized by flow recirculation and mixing with leakage flow, shroud passage vortex, and free step cavity vortex. The under cavity vortex, passage vortex, and leakage flow meet the blade wake, hence intensifying the losses. It seems that by incorporating a tip cavity, the leakage flow is reduced drastically about $25 \%$, as seen from Figure 12(a) compared to the case of a flat tip. Moreover, the flow recirculation in the tip cavity seems to reenergize the flow by a ventilation near the shroud, and subsequently the total-to-total and total-to-static isentropic efficiencies are increased by $5.4 \%$ and $13.26 \%$ as seen from Figure 12(b) and (Figure 12(c)), respectively.

3.2. Impacts of the Rotor Blade Positions. The effects of varying the pitchwise positions of the rotor blade on the expansion are assessed in terms of the total-to-total and totalto-static isentropic efficiencies and the pressure coefficient. The trigger positions $\left(4^{\circ}, 2^{\circ}, 0^{\circ},-2^{\circ}\right.$, and $\left.-4^{\circ}\right)$ are indicated 


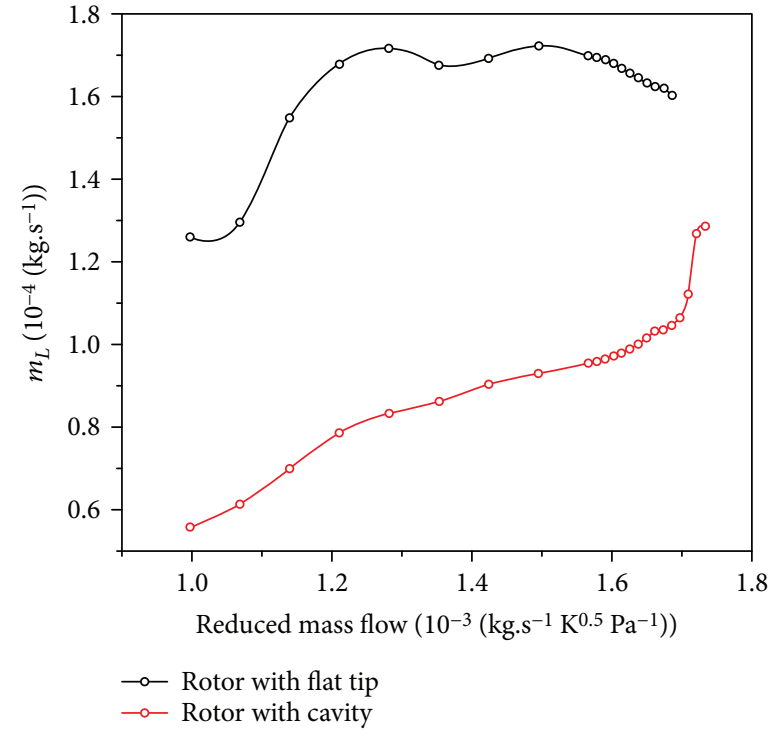

(a)

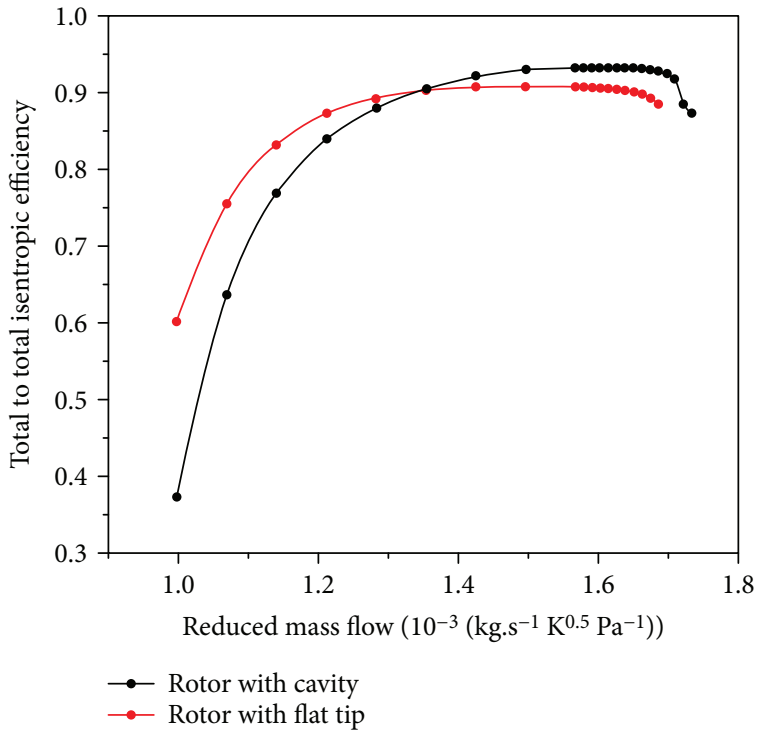

(b)

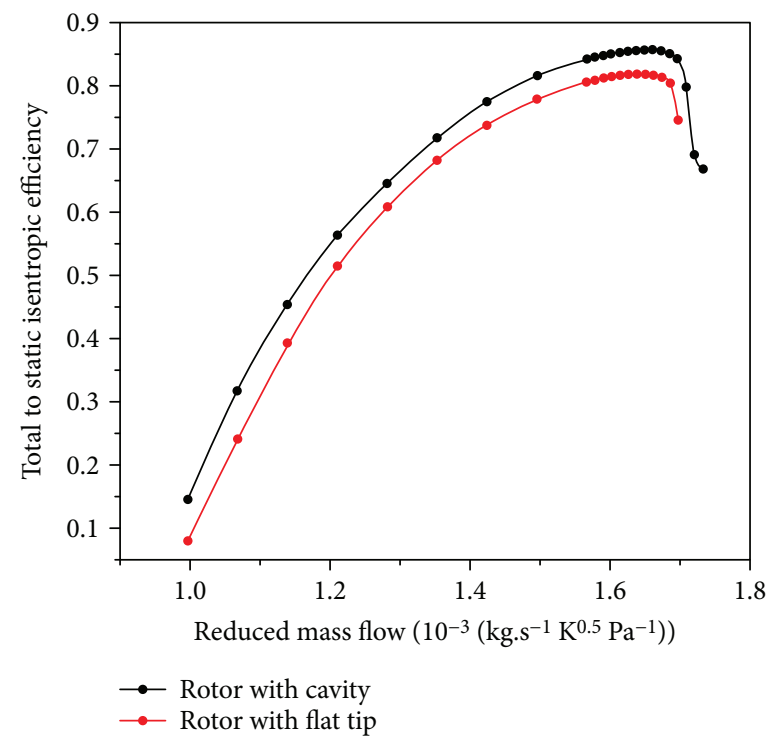

(c)

Figure 12: Comparison between flat tip and tip cavity: (a) leakage flow rate, (b) total-to-total isentropic efficiency, and (c) total-to-static isentropic efficiency.

by Figure 13. The relative variations of the total-to-total efficiency and total-to-static efficiency are defined as $100 \times\left(\eta / \eta_{0}-1\right)$, where $\eta_{0}$ is the efficiency at the trigger position R0, and presented in Figure 14 for different trigger positions and operating points. The peak values are reached for the positions of R1 and R4. The shift of efficiency curves is explained by the vane wake interacting with the blade leading edge. As observed, the minimum of isentropic efficiency occurs at the nominal operating point. When the wake meets the blade leading edge, and this represents the position of minimum losses.

The pressure coefficient $C_{p}=\left(P-P_{\text {exit }}\right) /(1 / 2) \check{\rho} \check{W}_{\text {exit }}^{2}$ over the vanes is practically invariable with the pitchwise positions, except near the trailing edge owing to the back pressure generated from the rotor blades. The plot of $C_{p}$ along the second blade exhibits (Figure 15) a predominant variation of pressure loading at the leading edge caused by incoming vane wake. Also, the sudden change at $50 \%$ of the blade chord seen from the suction side is referred to as a local lambda shock wave, which is more intense at the maximum mass flow as revealed by a drop in $C_{p}$ shifted towards the trailing edge from $75 \%$ of the chord. At the minimum mass flow rate, the noticeable variation in $C_{p}$ occurs with an opposite pressure gradient over the fore of the blade up to $20 \%$ of the chord. This can be explained by the displacement in stagnation point from the blade leading edge over the suction side, causing a negative incidence and creating a bubble recirculation over 


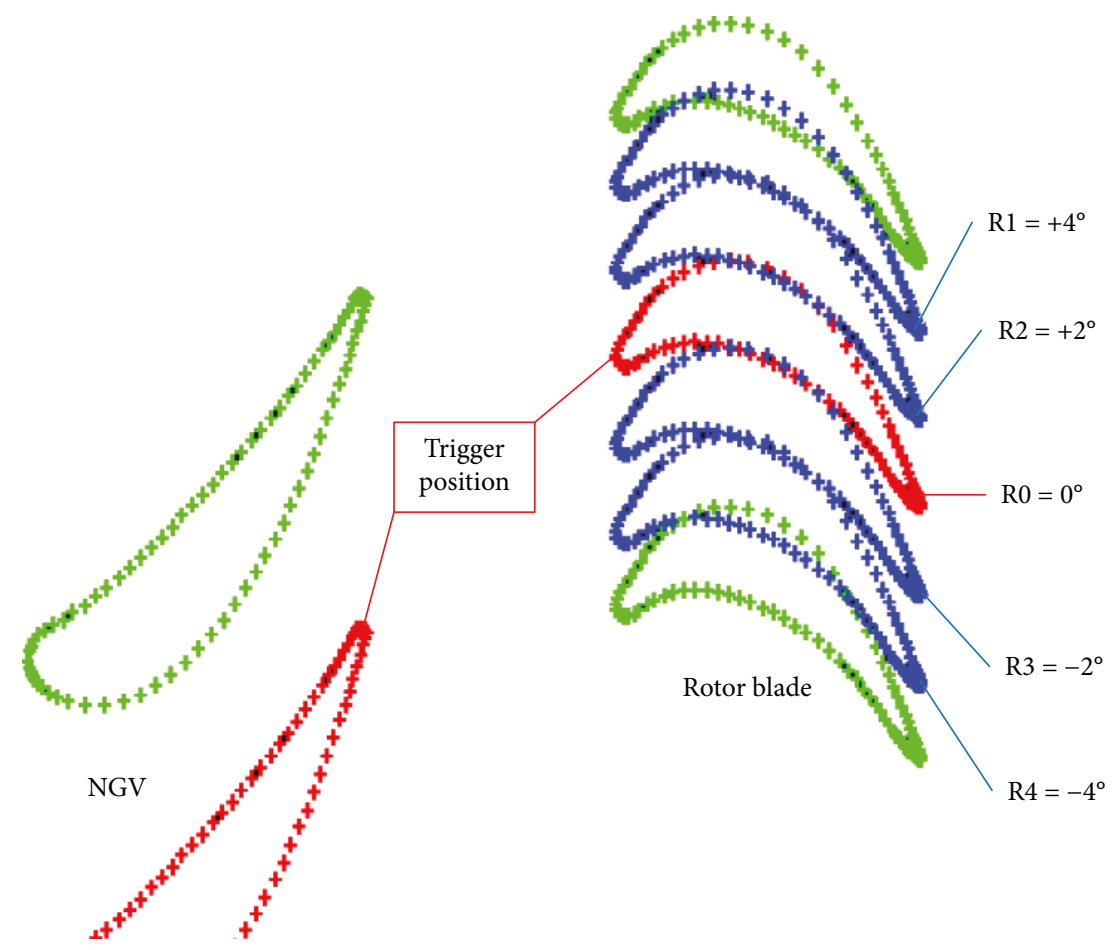

FIgURE 13: $2^{\text {nd }}$ blade trigger position.

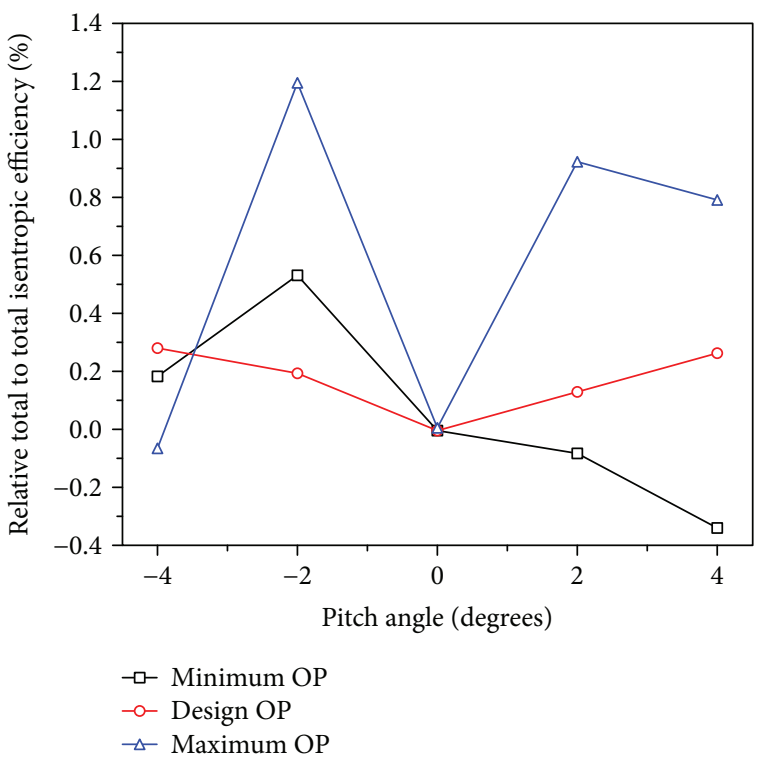

(a)

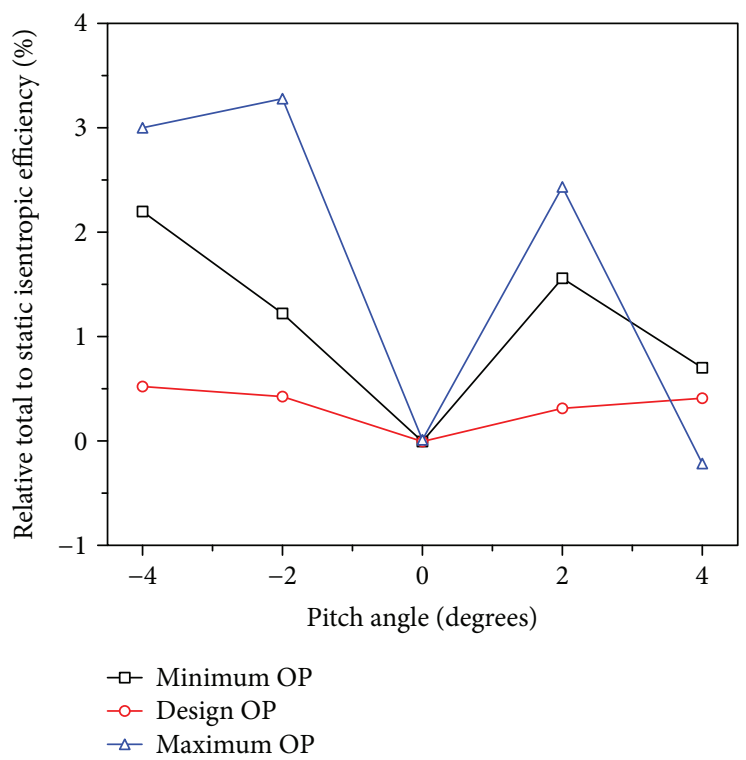

(b)

FIGURE 14: Effect of the pitchwise positions of rotor blade in terms of (a) total-to-total isentropic efficiency and (b) total-to-static isentropic efficiency.

the pressure side where an adverse pressure gradient occurs. As far as the pressure loading distribution is concerned, over the totality of rotor blade, the position leading to the maximum of turbine efficiency presents a wider area of static pressure distributions compared to that leading to the minimum of turbine efficiency, added to high $C_{p}$ at the leading edge related to the wakes segments impinging locally.

\section{Unsteady Flow Results}

The unsteady flow results are presented and discussed for the nominal operating point $(12,242 \mathrm{rpm}, m=69.5 \mathrm{~kg} / \mathrm{s})$. The time step was $\Delta t_{\min }=3.329 \mu \mathrm{s}$, and the total simulation time equal to $9.802 \mathrm{~ms}$ corresponding to two rounds of the rotor. However, by respecting the Shannon criteria [24], the period $\Delta t_{\min }$ was divided by 3 (equal to $1.019 \mu \mathrm{s}$ ) allowing detecting 

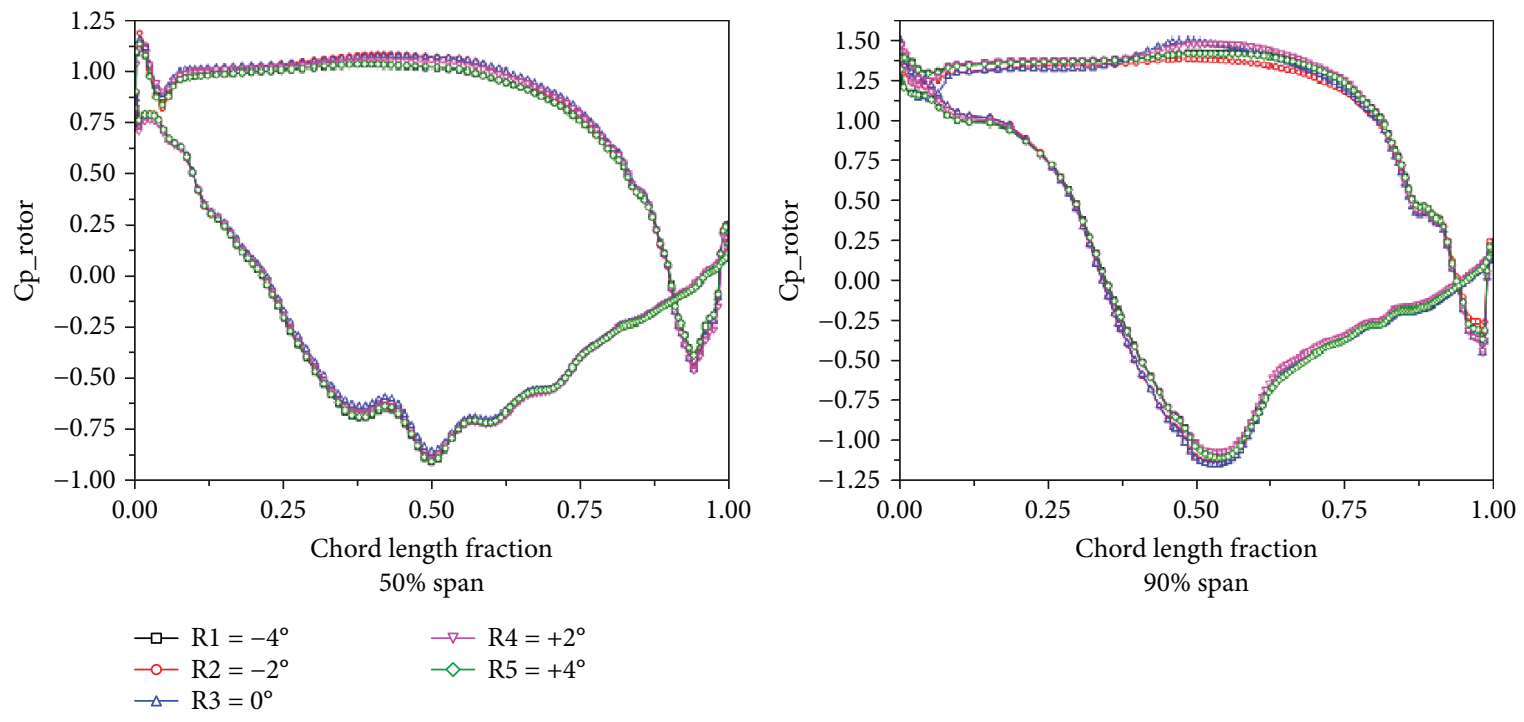

(a)
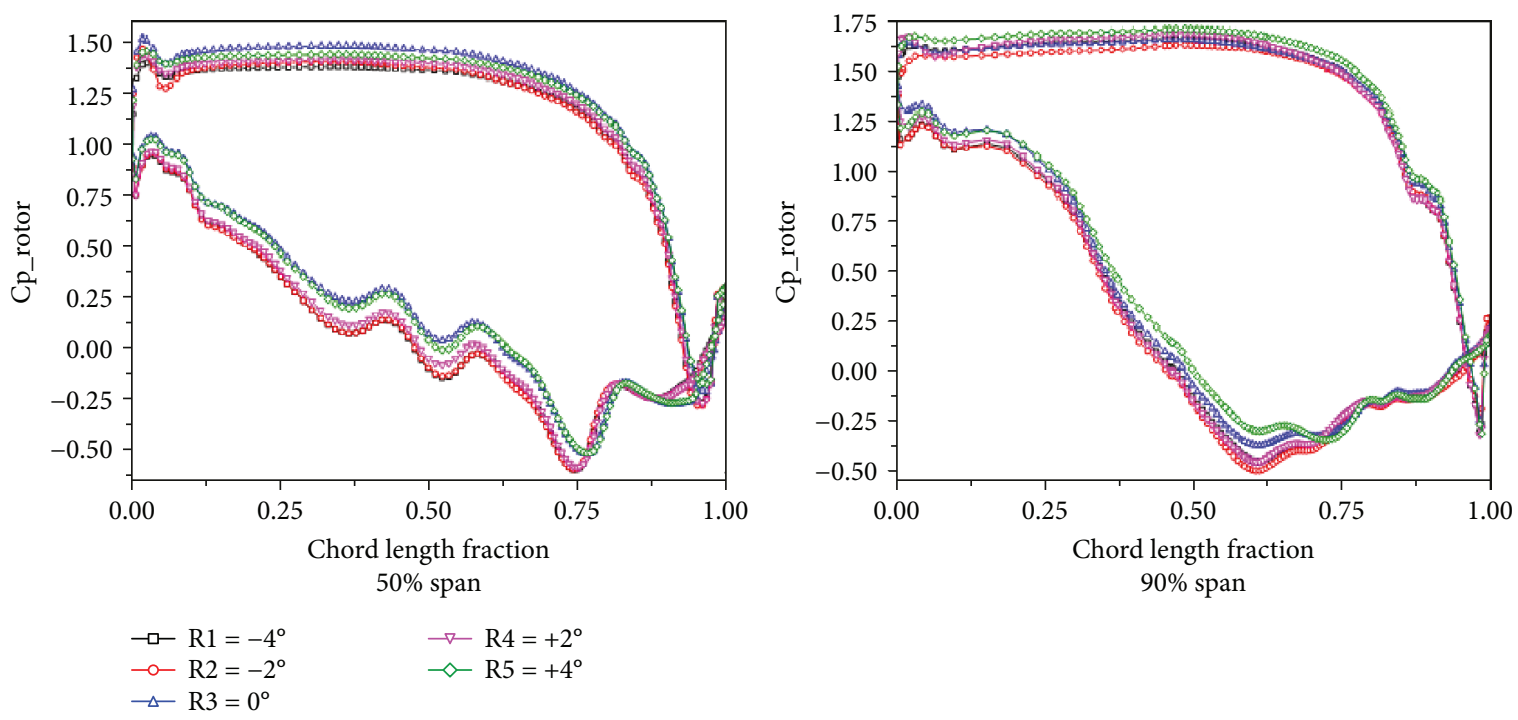

(b)

Figure 15: Pressure coefficient over the $2^{\text {nd }}$ blade at the different pitch positions and the (a) nominal operating point and (b) maximum mass flow rate operating point.

the small fluctuations of pressure. The recording points of temporal static pressure were at midpitch of first, second, and third vanes from $10 \%, 50 \%$, and $96 \%$ spans of the exit plane, whereas those upstream and downstream of the rotor blade, were set at the midpitch of first, second, third, and fourth blades. These positions were selected to better highlight the effect of flow structures: interactions of the vanes wakes and rotor blades and the vane shroud secondary flows with the blade tip leakage vortex, in addition to the vane hub secondary flow with the rotor hub secondary flow.

It was necessary to eliminate the transitory period from the temporal pressure signals as revealed from Figure 16. The recorded pressure signal is characterized by a first frequency of $100 \mathrm{~Hz}$ corresponding to the time of simulation which the magnitude is related by the fluctuations curve pace until its stabilization. Also, there are several peaks of

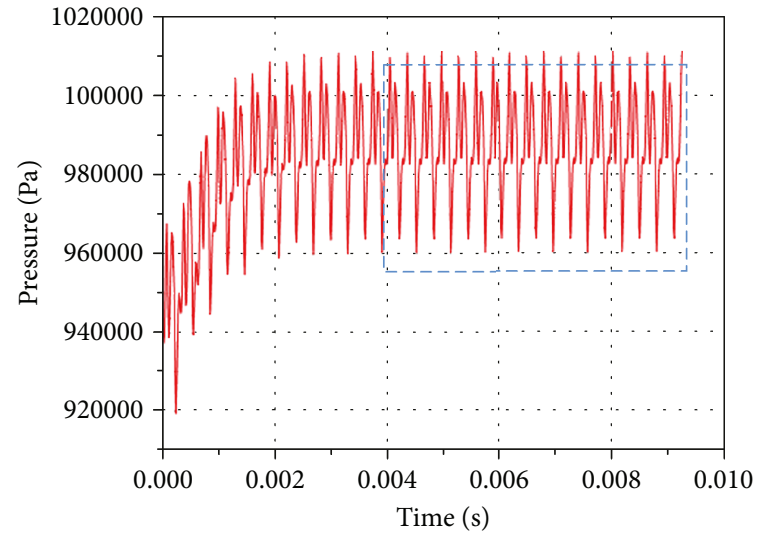

Figure 16: Sample of a temporal pressure signal. Stabilization: [4 ms, $9.8 \mathrm{~ms}$. 

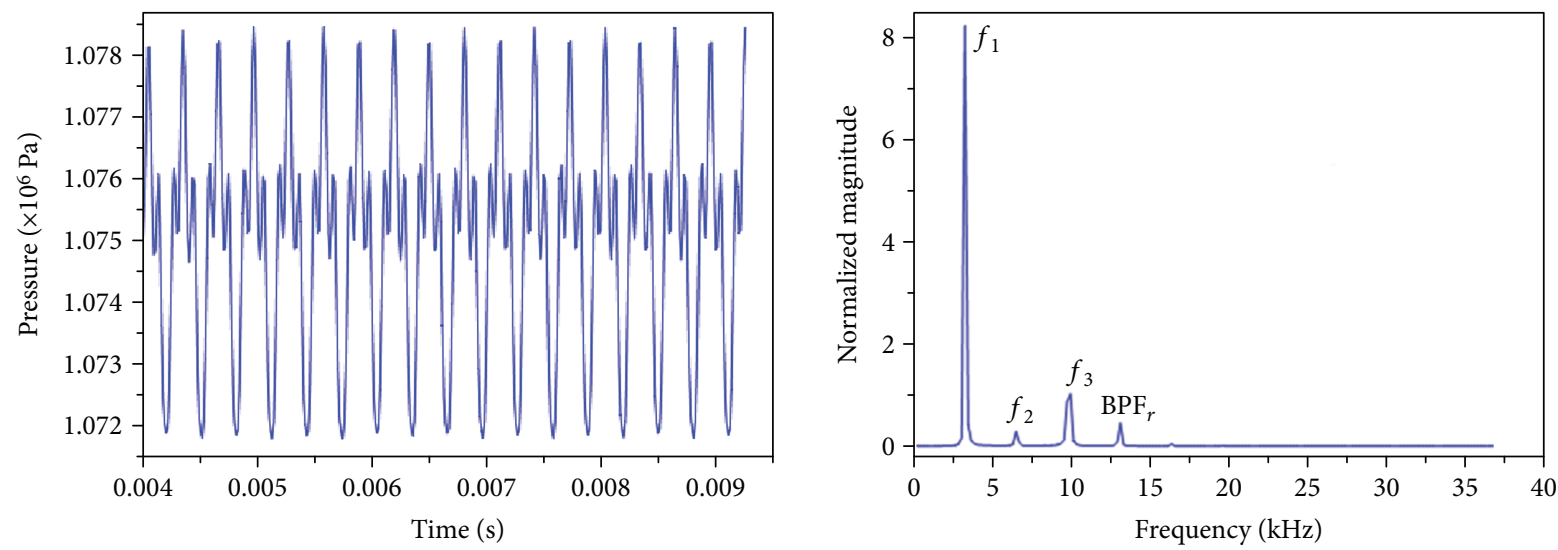

(a)
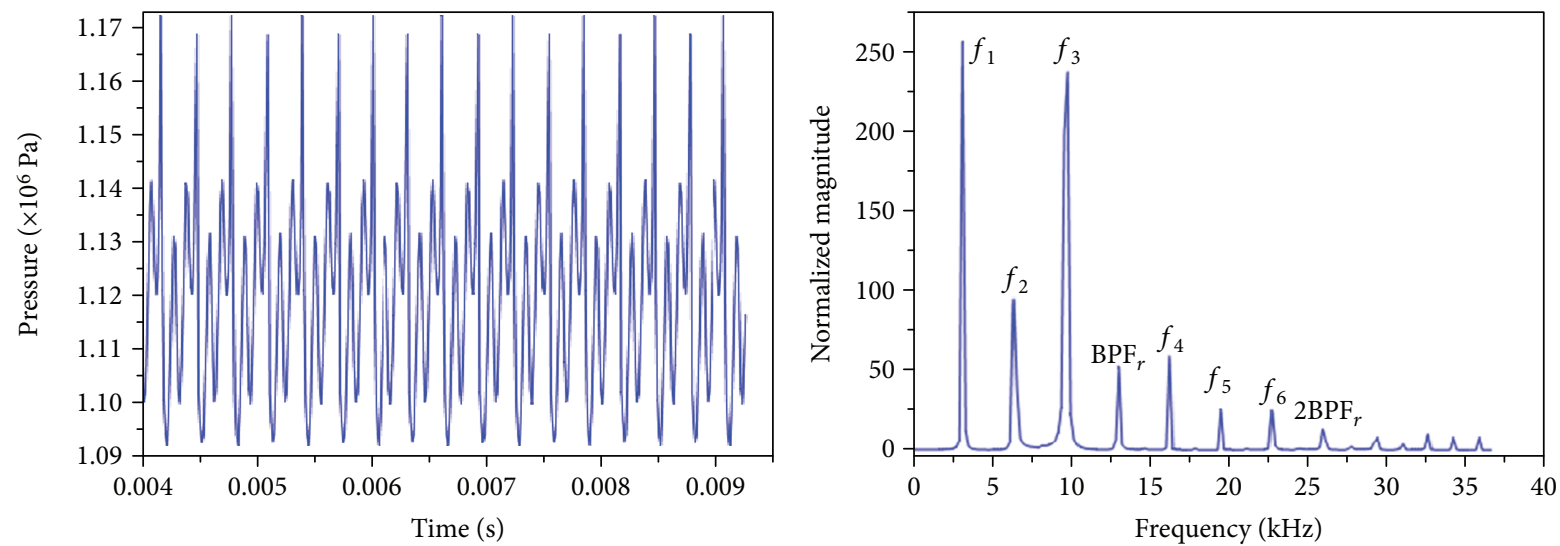

(b)
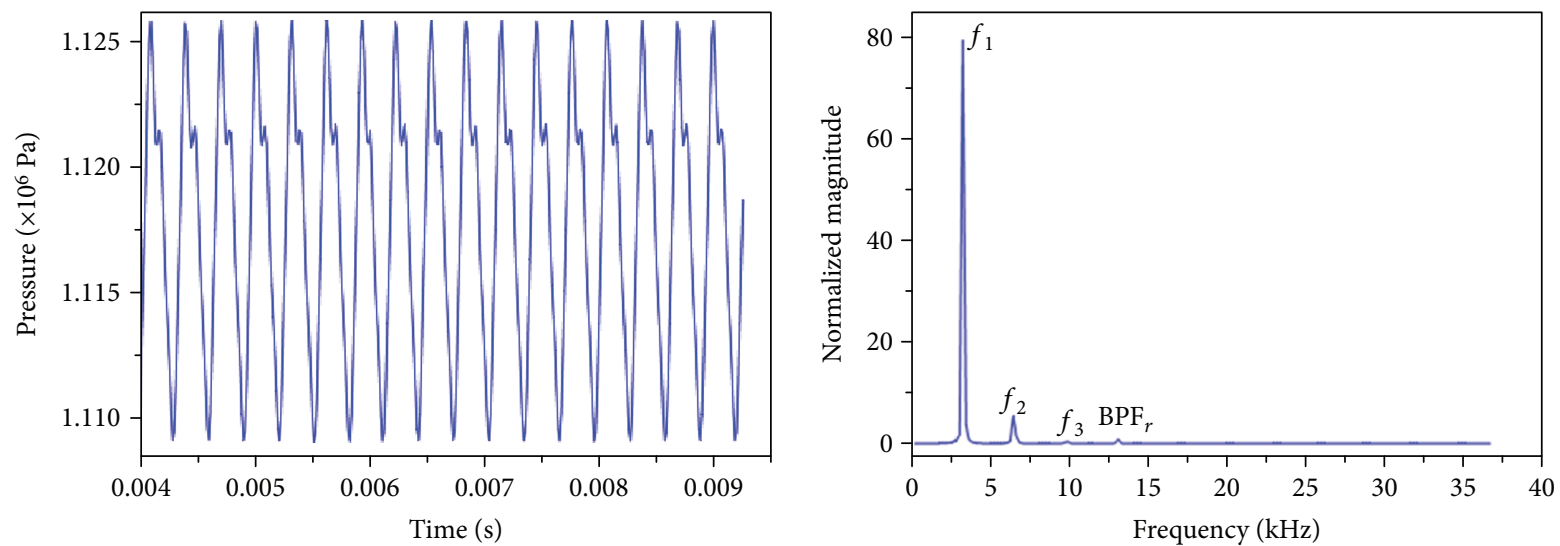

(c)

FIgURE 17: Temporal pressure fluctuations and FFT, recorded at the exit of NGV at midspan and midpitch of (a) first, (b) second, and (c) third vanes.

frequencies due to the transitory fluctuations of irregular variations with time (no fluctuation periodicity). The treated region in the time interval $4 \mathrm{~ms}-9.8 \mathrm{~ms}$, seems to have a regular periodicity in time and characterized by frequencies describing physical phenomena.

FFT analyses applied to the spatial and the temporal static pressures recorded at different positions and lines produced the spectrums in Figures 17-20, depict a fundamental blade passing frequency $\mathrm{BPF}=(\Omega / 60) N_{r}=13,066.68 \mathrm{~Hz}$ related to the potential effect of the rotor blades. The pressure waves characterized by this harmonic and its multiples propagate both upstream and downstream of blades and cause flow perturbations through the blade passage, but are attenuated throughout. The magnitudes of the principal peaks vary depending on the recording points as noticed for the same spanwise location. The wave amplitude seems to increase starting from the suction side until a maximum in the middle of interblade and then reduces until the pressure side. For this 

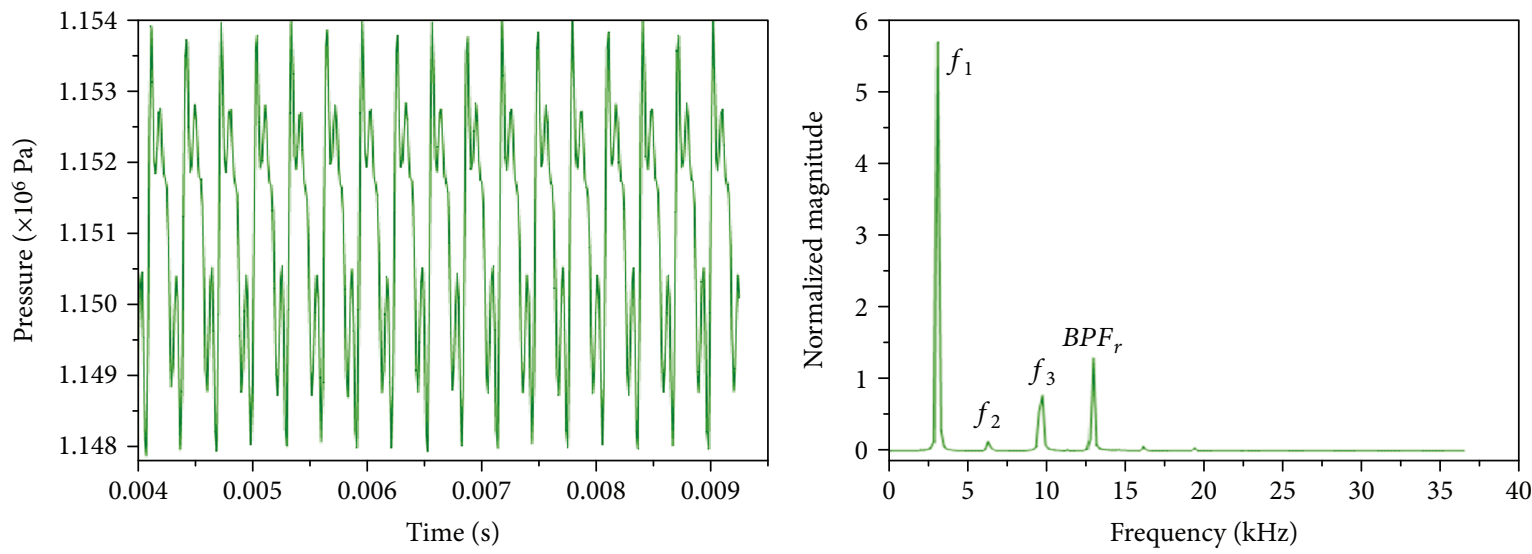

(a)
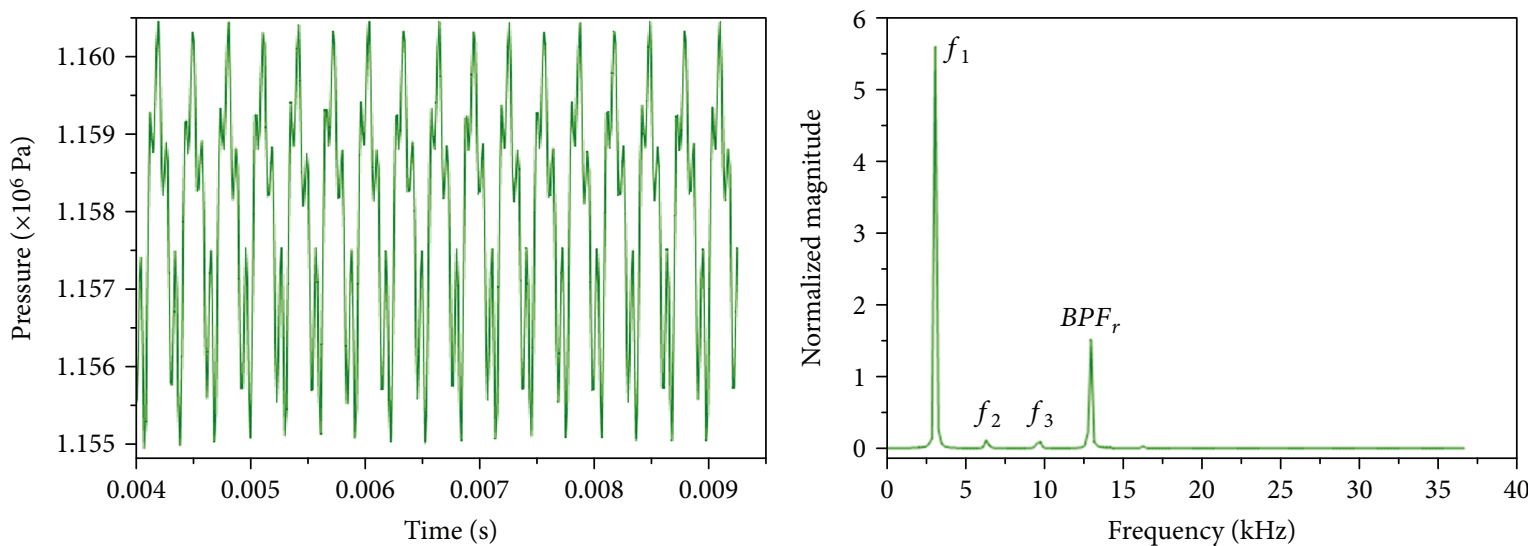

(b)
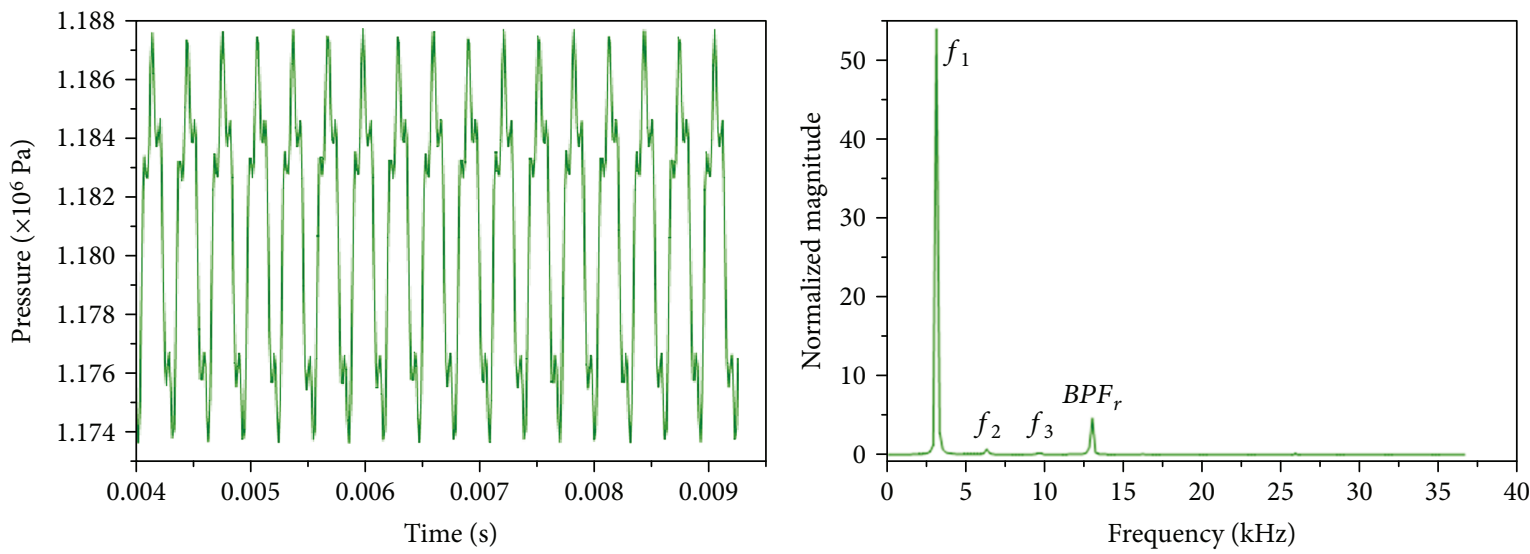

(c)

FIGURE 18: Temporal pressure fluctuations and FFT, recorded at the exit of NGV at the 96\% span and midpitch of (a) first, (b) second, and (c) third vanes.

reason, the temporal pressure signals were taken at the midpitch where they have the highest amplitudes. At the exit of vanes, the pressure fluctuations recorded at midspan and near shroud, specifically at the midpitch of first, second, and third vanes, and their associated spectrums are shown by Figures 17 and 18. Also appear other frequencies $f_{1}, f_{2}, f_{3}, f_{4}, f_{5}$, and $f_{6}$ of higher amplitudes attributed to the embedded rotor flow disturbances, predominately affected by upstream flow distortions caused by the vane wakes and the downstream potential disturbances related to the rotor blade, shroud ring and tip cavity. In principle, when two primary harmonics say, $f_{1}$ and $f_{2}$, coexist, the flow responses may contain subharmonics with frequencies like $n f_{1} \mp m f_{2}$, where $n$ and $m$ are integers. From one recording point to another, there is emergence of different frequency peaks of varying magnitudes which 


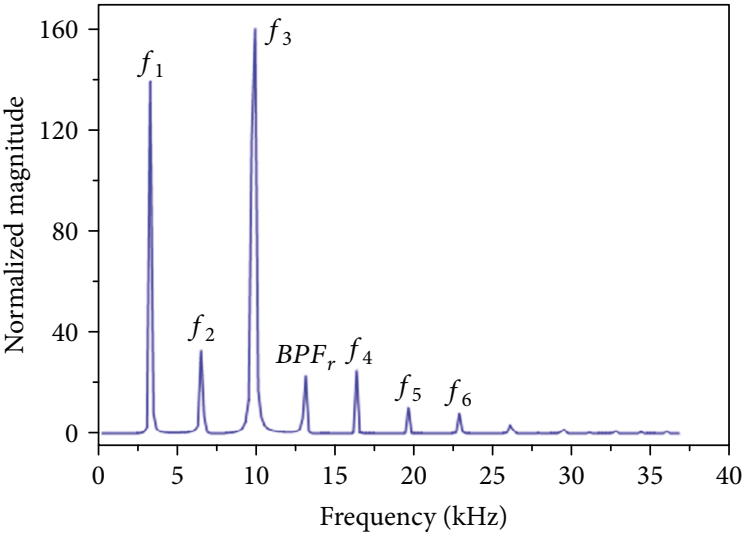

(a)

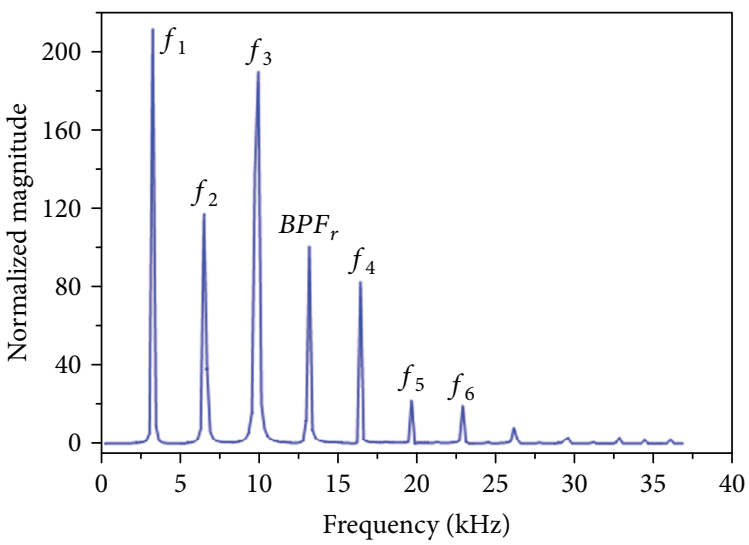

(c)

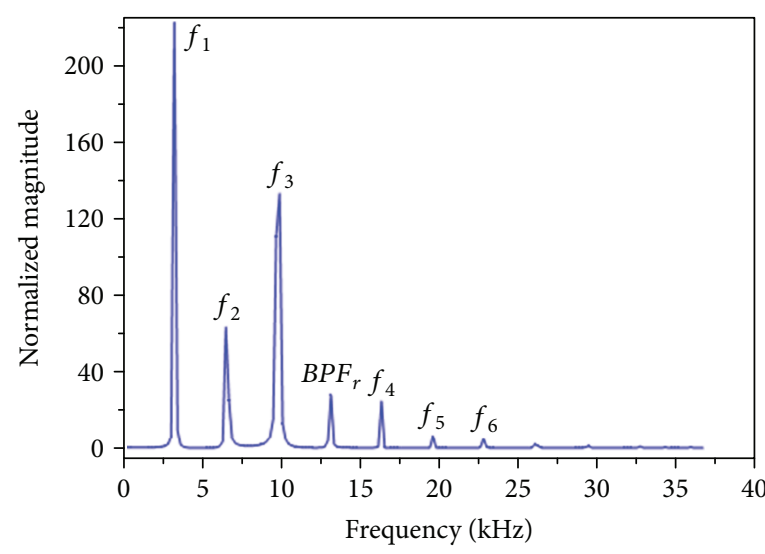

(b)

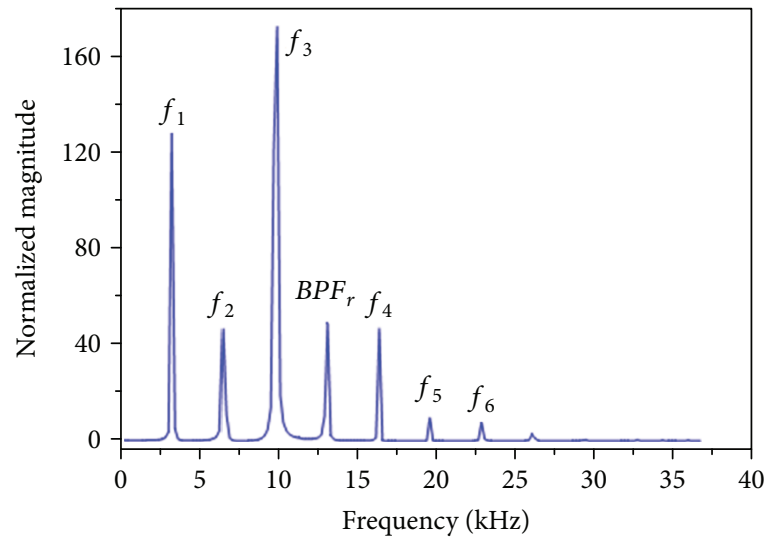

(d)

FIGURE 19: FFT of pressure fluctuations recorded upstream of the blade at (50\%) span and midpitch of (a) first, (b) second, (c) third, and (d) fourth blades.

depends on the relative blade position, since the blades do not coincide with the vanes' wakes at the same instant. At the recording point just behind the trailing edge of the vane, the magnitude of frequency $f_{1}$ reaches the maximum but lesser when the recording point is not aligned with the trailing edge. The magnitude of the frequency $f_{2}$ is lower than $f_{1}$ due to backward potential effect which has a magnitude weaker than that implied by the vanes' wakes. The frequencies $f_{2}$ and $f_{3}$ are more pronounced for the second vane recording point, added to other important frequencies $f_{4}, f_{5}$, and $f_{6}$. Figure 18 shows that when the static pressure was recorded near the shroud, the first frequency is still important whereas the others are less compared with BPF.

Figures 19 and 20 show the spectrums of pressure fluctuations recorded just upstream the blade leading edge, which indicate that the main frequencies of the temporal evolutions are stronger than downstream of vanes. The strength of the potential effects is such that BPF is felt upstream the blade leading edge; however, its magnitude is lesser than the first frequencies $f_{1}$ and $f_{2}$. The pressure fluctuations recoded upstream the rotor blade is characterized by more peaks of frequencies as described by the chorochronic theory and revealed by FFT analysis. The magnitudes of the fundamental frequencies depend on the recoding locations which are more significant at the midspan than hub and shroud. Moreover, the frequencies such as $f_{1}, f_{2}, f_{3}, f_{4}, f_{5}, f_{6}$, and $\mathrm{BPF}$ have the highest amplitudes for the recording point of the third blade channel. Downstream of rotor blade, the temporal evolutions of static pressure are dominated by the composition of the frequencies $f_{1}$ and $f_{2}$. The vanes wakes are chopped by the rotor blades and convected downstream until the rotor exit and afterwards decay. The frequency $f_{1}$ has a smaller magnitude near the hub as might be explained by the formation of hub vortex convected towards the rotor exit. Moreover, high amplitudes frequency peaks are seen near the shroud for the blades 3 and 4, and hence, the pressure fluctuations induced by the tip cavity vortices are of higher amplitudes than the convected wakes or the hub passage vortices.

The distributions of static pressure and entropy at the instant of $5.17 \mathrm{~ms}$ are shown by Figures 21 and 22 for different normalized axial distances. These parameters are independent of the frame of reference, and their azimuthal evolutions are continuous across the interfaces. The static pressure contours (Figure 21) highlight the potential effect of the rotating blades as confirmed by the shape of the pressure wave, which increases close to the blade leading edge. 


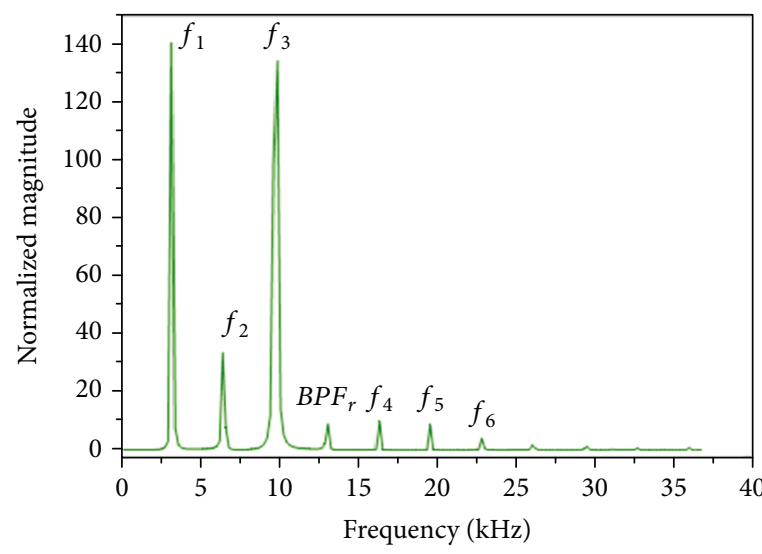

(a)

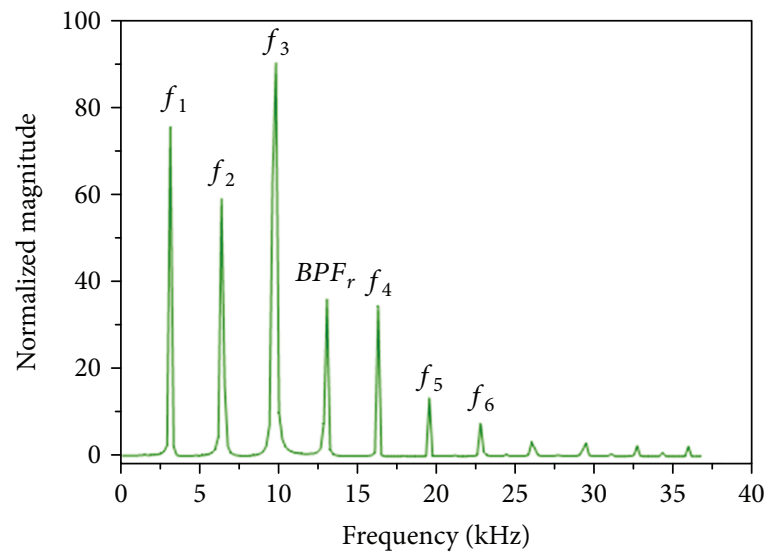

(c)

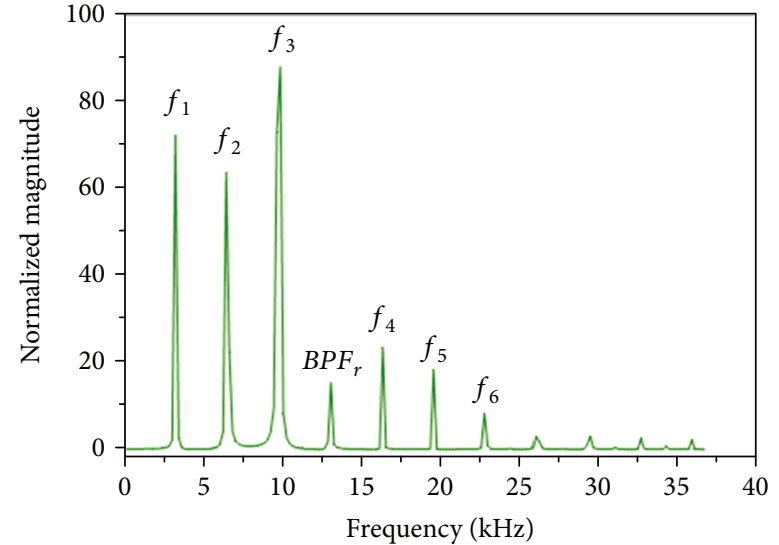

(b)

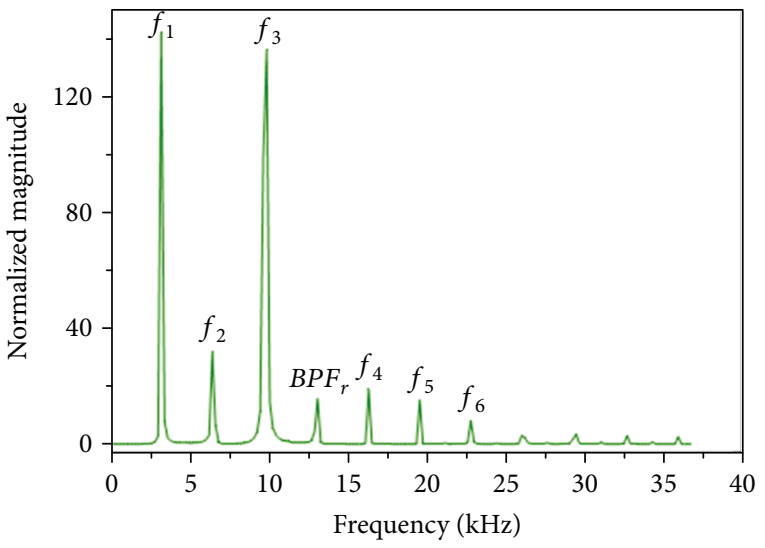

(d)

Figure 20: FFT of pressure fluctuations recorded upstream of the blade at (96\%) span and midpitch of (a) first, (b) second, (c) third, and (d) fourth blades.

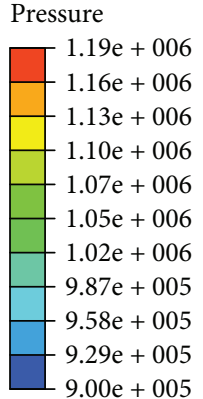

$(\mathrm{Pa})$

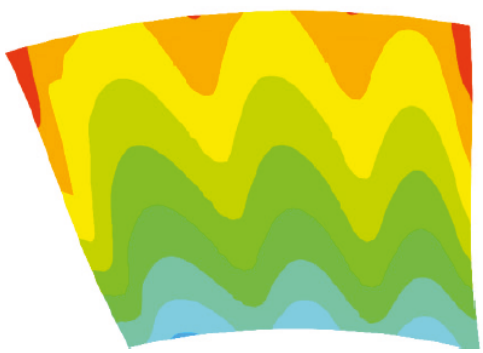

0.97

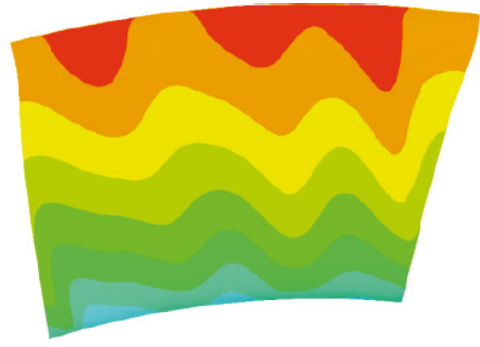

1.05

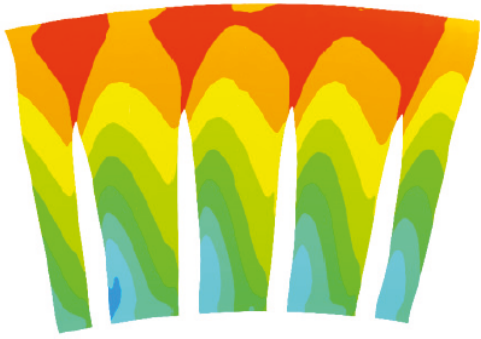

1.25

FIGURE 21: Static pressure contours at different normalized streamwise cross-sections, at the instant of $5.17 \mathrm{~ms}$.

Downstream of rotor blade, there are 64 lobed structures with different forms of pressure waves, related to the vane wakes chopped by the rotor blades, of a decaying amplitude owing to mixing. These results are also confirmed by the contours of entropy (Figure 22) highlighting regions of vanes wakes in addition to secondary flows (hub and shroud passage vortices ...) convected downstream. The entropy distributions near the blade leading edge show evidence of losses related to VRI which produce a circumferential distortions with a clear deficit in flow velocity. Downstream of rotor blade, the dominant losses are those related to the leakage flow and the convected wakes and secondary flows.

FFT analyses of spatial pressure fluctuations at the instant of $5.17 \mathrm{~ms}$ obtained from the recorded lines passing near hub, midspan, and shroud (96\%) are depicted in 


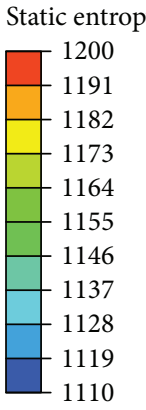

$\left(\mathrm{J} \mathrm{kg}^{-1} \mathrm{~K}^{-1}\right)$

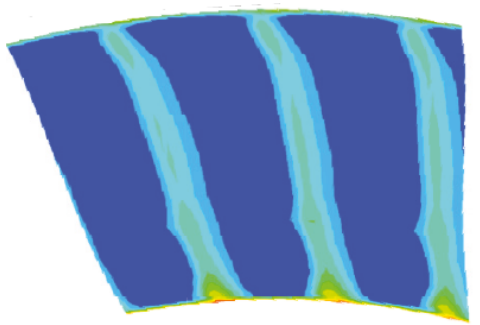

0.97

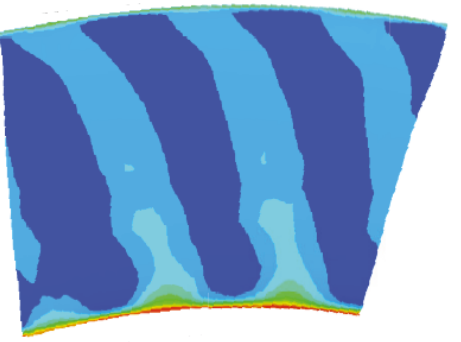

1.05

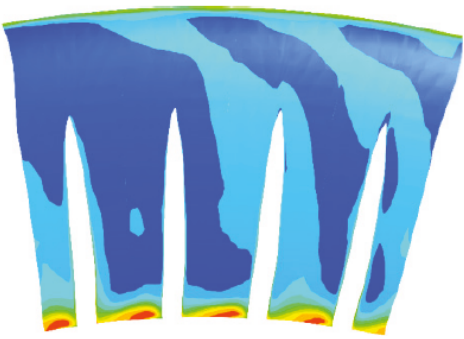

1.25

FIGURE 22: Entropy contours at different normalized streamwise cross-sections, at the instant of $5.17 \mathrm{~ms}$.
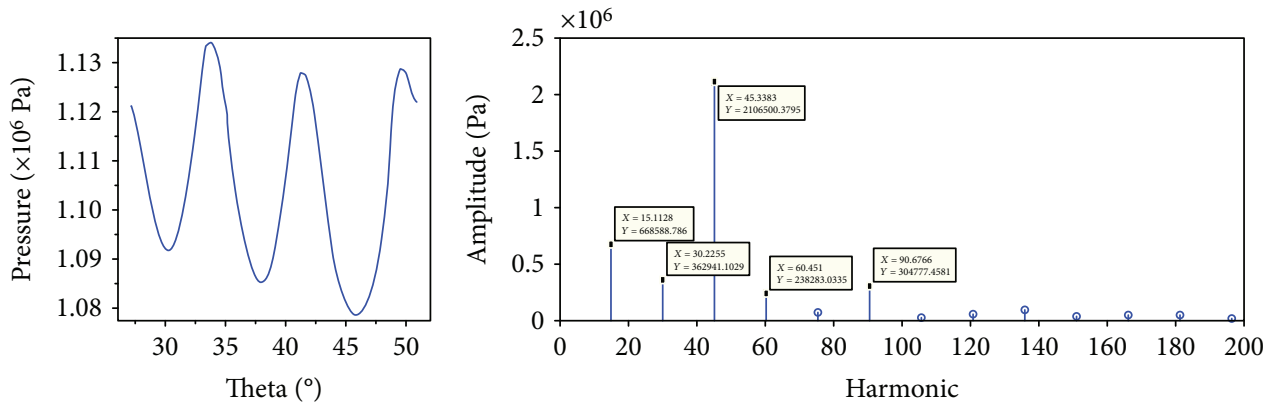

(a)
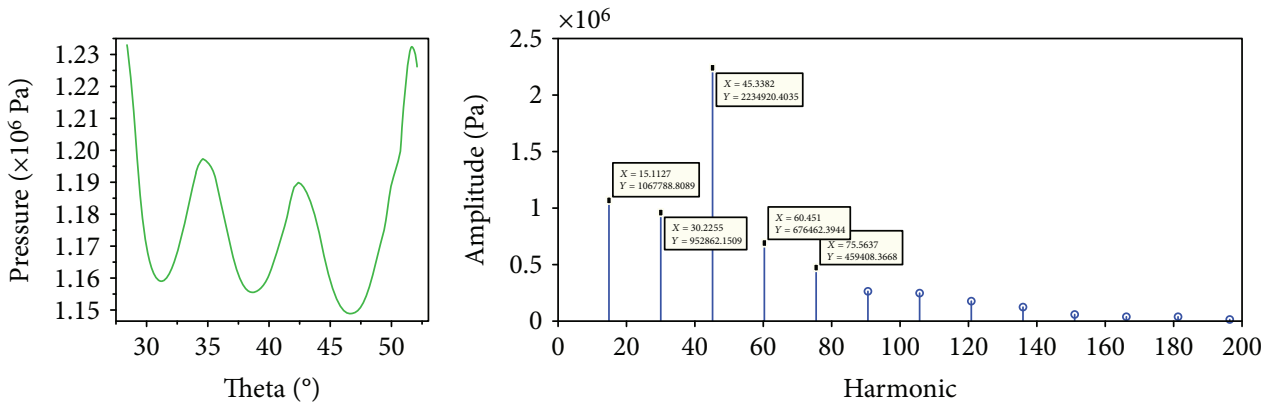

(b)

FIGURE 23: Spatial pressure distributions and FFT, recorded at the vane exit: (a) 50\% span and (b) 96\% span.

Figures 23-25. Indeed, the harmonic zero was eliminated since it represents the meridional distribution (average field) and qualifies the decay in the static pressure amplitude from inlet to outlet. The flow interactions are composed by an infinite number of rotating lobed structures described by Tyler and Sofrin [25], and given by $k=m N_{r}+n N_{s}$ and $n=\cdots,-1$, $0,1, \ldots$ of rotating speeds $\Omega_{k}=\left(m N_{r} / k\right) \Omega_{r}$. The interaction produced by the run of rotor blades in front of NGV produces the space modes resulting from the combination of vane count and blade count. As noticed from the respective FFT spectrums, the harmonics of $k=15.031,15.112$, $15.765, \ldots$ are approximated by the fundamental harmonic 16 and its multiples related to the stroboscopic effect induced by the passing of the blade row in the front of NGV. In this picture, the dominant unsteady fluctuation is due to the rotor trace itself. A physical illustration of this mechanism can be made, for example, the wakes coming from the vanes are characterized circumferentially by the spatial periodicity of $k=N_{n}$, and the potential effect of the rotating row is represented by the spatial periodicity of $k=N_{r}$. Thus, the wake/ potential interaction is being translated into nonlinear terms of $k=m N_{r} \mp n N_{n}$.

The spatial static pressure recorded along the lines passing by the midspan rear the vane trailing edge and their subsequent spectrums are depicted in Figure 23 showing clearly the dominant harmonic 48 corresponding to NGV wakes. The vane and blade counts present a common divider, explaining the appearance of the harmonic 16 related to the first order combination between the vane and blade counts. The presence of the spatial harmonic 64 is the revelation of the potential effect which is of smaller amplitude compared with the fundamental harmonics downstream of vanes. On the contrary, close to the rotor blade (Figure 24), the amplitude of the harmonic 64 increases as the potential effect 

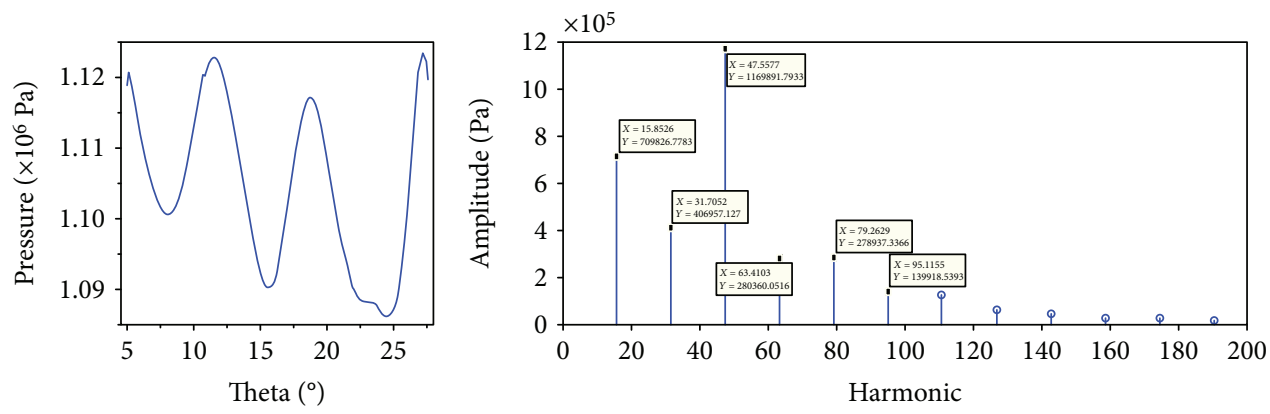

(a)
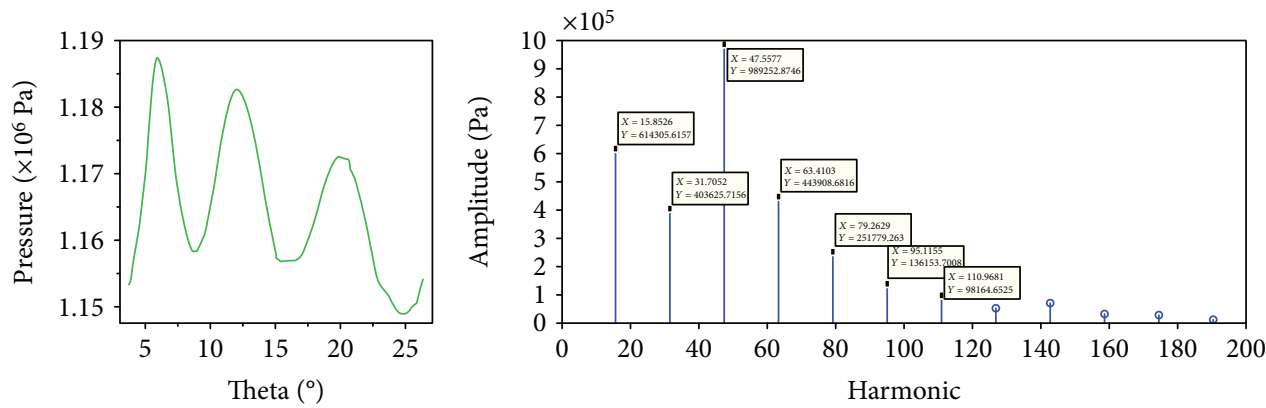

(b)

FIGURE 24: Spatial pressure and FFT, recorded upstream rotor at lines: (a) 50\% span and (b) 96\% span.
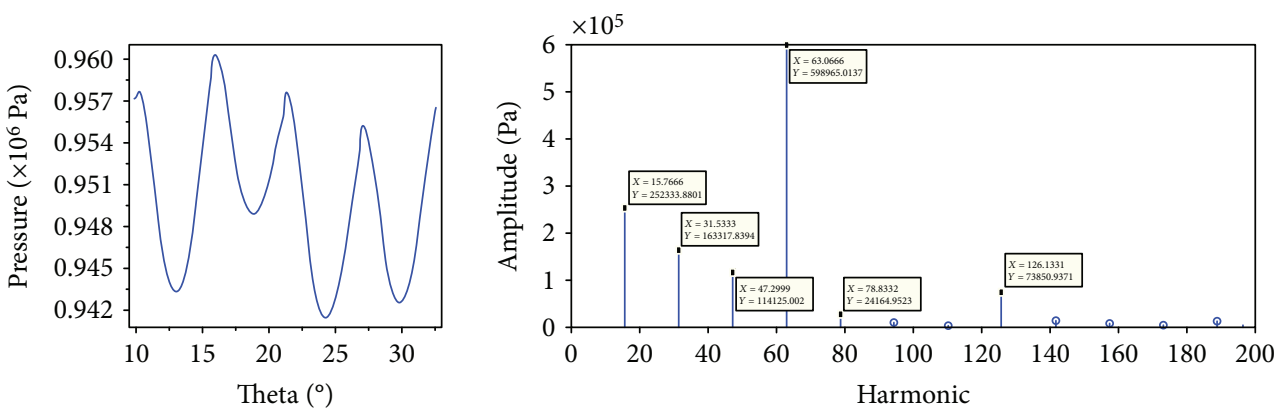

(a)
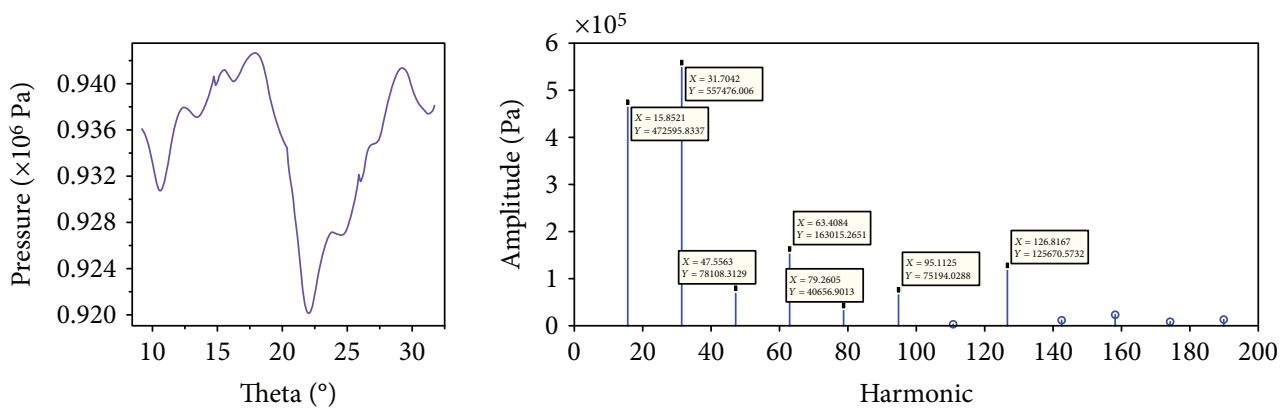

(b)

Figure 25: Spatial pressure and FFT, recorded downstream rotor blades at lines: (a) 50\% span and (b) $96 \%$ span.

becomes more significant. Through the blade row, the flow undergoes sudden azimuthal and radial pressure gradients and sustains the effects of vanes' wake segments interacting with the potential effect. The accumulation of these various mechanisms contributes to a radial mixing and an exchange of energy. Downstream of rotor blade, the spectrums of the recorded pressure fluctuations (Figure 25) exhibit the fundamental harmonic 64 and its multiples which are the 
spatial modulation of the rotor blades and represent in the first order the rotor blade wakes but later they decay due to mixing. A second spatial harmonic is superimposed which is a combination of the first order between the vane and blade counts. The incoming wakes and vortices are shown to persist downstream of the rotor but of attenuated amplitudes. Moreover, the fluctuations induced by the tip cavity vortices are more significant than the wakes or the hub passage vortices.

The simulations based on the reduced domain NGV $3: 4$ allowed detecting the modes with harmonics less than the vane and blade counts. The harmonic 48 represents the effect of the vanes' wakes chopped by rotor blades, whereas the harmonic 64 represents the potential effect of rotating blades, while the harmonic 16 represents the VRI. Downstream of $\mathrm{NGV}$, the effects of wakes are dominant, but the potential effect increases close to the rotor blades. Their effects are pronounced along the spacing between vanes and blades but attenuate downstream. From other unsteady simulations which considered a single channel by a component, in addition to overestimating the pressure fluctuation the frequencies of interactions related to the effects of wake segments chopped by rotor blades and other vortex structures were not detected.

\section{Conclusion}

The reduced computational domain NGV 3:4 seems a compromised choice to perform both the steady and unsteady flow computations. The flow structures in the vanes and the shrouded rotor with a tip cavity were characterized in details. As shown the wakes and secondary flows from the vanes persist downstream the rotor. Unsteady flow simulations based on the transient rotor/stator interface permitted investigating the unsteady VRI which are the valuable data in analyzing the forced vibrations. The amplitudes of the temporal pressure fluctuations due to rotor blade passing events and their harmonics vary significantly with the probing locations. The circumferential distribution of static pressure in each component reveals the existence of a unique harmonic and its multiples, related to the vane/blade count. The main interaction contibution is that due to the potential effect which propagates in the form of pressure wave upstream and downstream of the blade row. The other forms of VRI seem attributed to the embedded rotor flow disturbances predominately affected by the wake distortions impinging on the rotor blade suction surface, added to the shroud and tip cavity disturbances and the vortices formation of lesser importance.

\section{Nomenclature}

$\begin{array}{ll}C: & \text { Blade chord }(\mathrm{m}) \\ C_{p}: & \text { Pressure coefficient } \\ f: & \text { Frequency }(\mathrm{Hz}) \\ h: & \text { Enthalpy }(\mathrm{J} / \mathrm{kg}) \\ k: & \text { Turbulence kinetic energy }\left(\mathrm{m}^{2} / \mathrm{s}^{2}\right) \\ \dot{m}: & \text { Mass flow rate }(\mathrm{kg} / \mathrm{s}) \\ n: & \text { Rotating speed }(\mathrm{rpm}) \\ N: & \text { Vane/blade count }\end{array}$

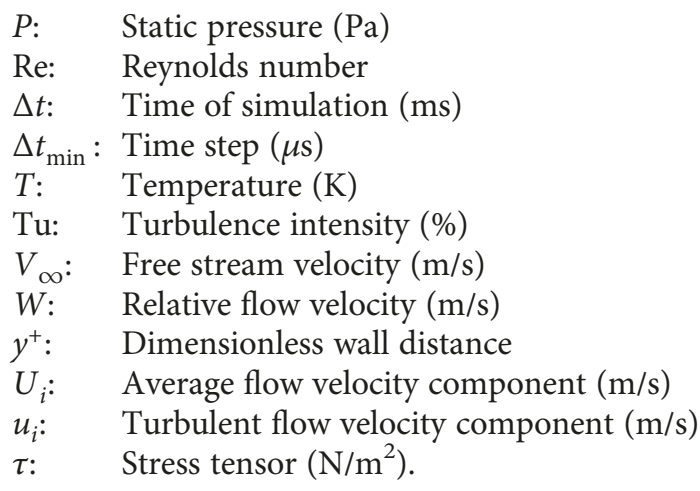

Greek

$\varepsilon:$ Turbulence rate of dissipation $\left(\mathrm{m}^{2} / \mathrm{s}^{3}\right)$

$\eta$ : Efficiency

$\mu$ : Dynamic viscosity $(\mathrm{kg} / \mathrm{m} \cdot \mathrm{s})$

$\rho$ : Density $\left(\mathrm{kg} / \mathrm{m}^{3}\right)$

$\omega$ : Specific turbulence dissipation rate.

\section{Subscripts}

$\begin{array}{ll}\text { is: } & \text { Isentropic } \\ n: & \text { Nominal } \\ t: & \text { Total } \\ \text { tt: } & \text { Total-to-total } \\ \text { red: } & \text { Reduced } \\ \mathrm{xt}: & \text { Transition. }\end{array}$

\section{Acronyms}

BPF: Blade passing frequency

hp: High pressure

NGV: Nozzle guide vane

VRI: Vane-rotor interactions

SST: Sheer stress transport.

\section{Data Availability}

The data used to support the findings of this study are included within the article.

\section{Conflicts of Interest}

The author declared no potential conflicts of interest with respect to the research, authorship, and/or publication of this article.

\section{References}

[1] M. B. Giles, "Calculation of unsteady wake/rotor interaction," in 25th AIAA Aerospace Sciences Meeting, Reno, Nevada, USA, March 1987.

[2] H. P. Hodson, "Measurements of wake-generated unsteadiness in the rotor passages of axial flow turbines," Journal of Engineering for Gas Turbines and Power, vol. 107, no. 2, pp. 467475, 1985.

[3] R. E. Walraevens, H. E. Gallus, A. R. Jung, J. F. Mayer, and H. Stetter, "Experimental and computational study of the unsteady flow in a 1.5 stage axial turbine with emphasis on 
the secondary flow in the second stator," in ASME 1998 International Gas Turbine and Aeroengine Congress and Exhibition: Volume 1: Turbomachinery, Stockholm, Sweden, June 1998.

[4] O. P. Sharma, E. Renaud, T. L. Buttler, K. Milsaps Jr., R. P. Dring, and H. D. Joslyn, "Rotor-stator interaction in multistage axial-flow turbines," in 24th Joint Propulsion Conference, Boston, MA, USA, July 1988.

[5] A. Binder, W. Förster, H. Kruse, and H. Rogge, "An experimental investigation into the effect of wakes on the unsteady turbine rotor flow," Journal of Engineering for Gas Turbines and Power, vol. 107, no. 2, pp. 458-465, 1985.

[6] O. P. Sharma, T. L. Butler, H. D. Joslyn, and R. P. Dring, "Three-dimensional unsteady flow in an axial flow turbine," Journal of Propulsion and Power, vol. 1, no. 1, pp. 29-38, 1985.

[7] N. Arndt, "Blade row interaction in a multistage low-pressure turbine," Journal of Turbomachinery, vol. 115, no. 1, pp. 137146, 1993.

[8] R. J. Miller, R. W. Moss, R. W. Ainsworth, and N. W. Harvey, "The development of turbine exit flow in a swan-necked interstage diffuser," in ASME Turbo Expo 2003, collocated with the 2003 International Joint Power Generation Conference: Volume 6: Turbo Expo 2003, Parts A and B, Atlanta, Georgia, USA, June 2003.

[9] V. S. P. Chaluvadi, A. I. Kalfas, H. P. Hodson, H. Ohyama, and E. Watanabe, "Blade row interaction in a high-pressure steam turbine," Journal of Turbomachinery, vol. 125, no. 1, pp. 1424, 2003.

[10] J. Schlienger, A. I. Kalfas, and R. S. Abhari, "Vortex-wakeblade interaction in a shrouded axial turbine," Journal of Turbomachinery, vol. 127, no. 4, pp. 699-707, 2005.

[11] L. Qi and Y. Zhou, "Turbine blade tip leakage flow control by unsteady periodic wakes of upstream blade row," Procedia Engineering, vol. 80, pp. 202-215, 2014.

[12] M. B. Giles, "Calculation of unsteady wake/rotor interaction," Journal of Propulsion and Power, vol. 4, no. 4, pp. 356-362, 1988.

[13] M. M. Rai and N. K. Madavan, "Multi-airfoil Navier-Stokes simulations of turbine rotor-stator interaction," Journal of Turbomachinery, vol. 112, no. 3, pp. 377-384, 1990.

[14] A. Arnone and R. Pacciani, "Rotor-stator interaction analysis using the Navier-Stokes equations and a multi-grid method," Journal of Turbomachinery, vol. 118, no. 4, pp. 679-689, 1996.

[15] J. P. Clark, G. M. Stetson, S. S. Magge, R. H. Ni, C. W. Haldeman Jr., and M. G. Dunn, "The effect of airfoil scaling on the predicted unsteady loading on the blade of a 1 and $1 / 2$ stage transonic turbine and a comparison with experimental results," in ASME Turbo Expo 2000: Power for Land, Sea, and Air: Volume 1: Aircraft Engine; Marine; Turbomachinery; Microturbines and Small Turbomachinery, Munich, Germany, May 2000.

[16] J. Yao, R. L. Davis, J. J. Alonso, and A. Jameson, "Massively parallel simulation of the unsteady flow in an axial turbine stage," Journal of Propulsion and Power, vol. 18, no. 2, pp. 465-471, 2002.

[17] JT8D-17A, Turbofan Engine, SPEC. NO. 6281, Date: 6-27-80, Pratt \& Whitney Aircraft group, 1980.

[18] Ansys-CFX, Theory Guide. Release 16.0, ANSYS, Inc., Canonsburg, USA, 2015.

[19] D. A. Wilcox, "Simulation of transition with a two-equation turbulence model," AIAA Journal, vol. 32, no. 2, pp. 247255, 1994.
[20] P. Adami and F. Martelli, "Three-dimensional unsteady investigation of HP turbine stages," Proceedings of the Institution of Mechanical Engineers, Part A: Journal of Power and Energy, vol. 220, no. 2, pp. 155-167, 2006.

[21] C. Zhou, H. Hodson, and C. Himmel, "The effects of trailing edge thickness on the losses of ultrahigh lift low pressure turbine blades," Journal of Turbomachinery, vol. 136, no. 8, article 081011, 2014.

[22] Z. Li, Z. Wang, X. Wei, and D. Qin, "Flow similarity in the rotor-stator interaction affected region in prototype and model Francis pump-turbines in generating mode," Journal of Fluids Engineering, vol. 138, no. 6, article 061201, 2016.

[23] R. E. Mayle, "The 1991 IGTI scholar lecture: the role of laminar-turbulent transition in gas turbine engines," Journal of Turbomachinery, vol. 113, no. 4, pp. 509-536, 1991.

[24] C. E. Shannon, "Communication in the presence of noise," Proceedings of the IEEE, vol. 86, no. 2, pp. 447-457, 1998.

[25] J. M. Tyler and T. G. Sofrin, "Axial flow compressor noise studies,” SAE Transactions, vol. 70, pp. 309-332, 1962. 


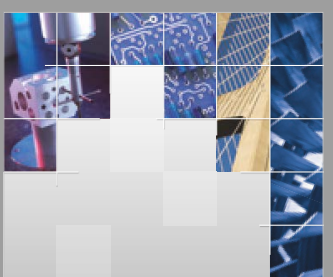

\section{Enfincering}
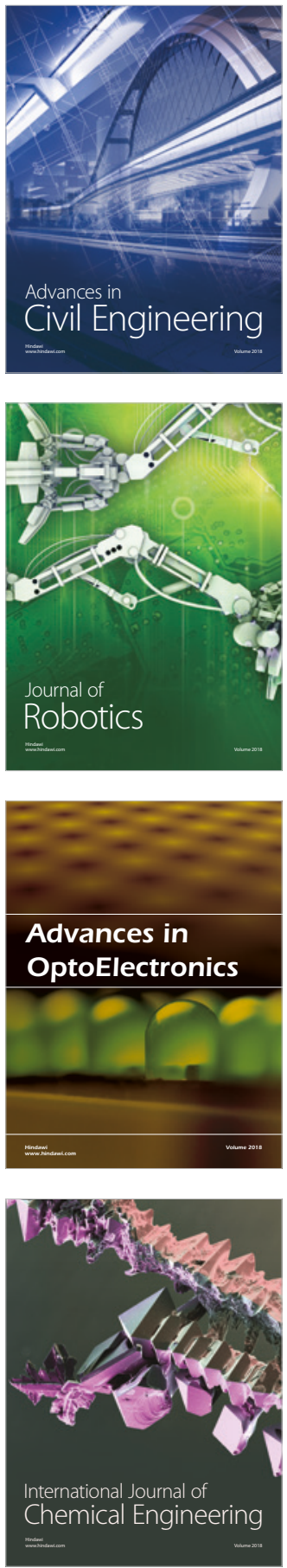

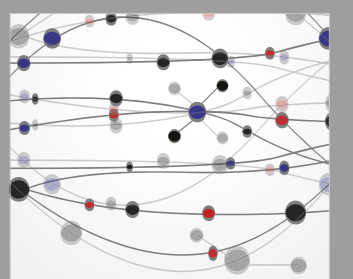

\section{Rotating \\ Machinery}

The Scientific World Journal

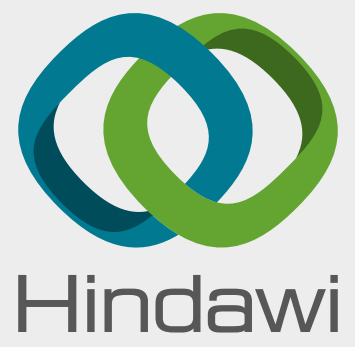

Submit your manuscripts at

www.hindawi.com
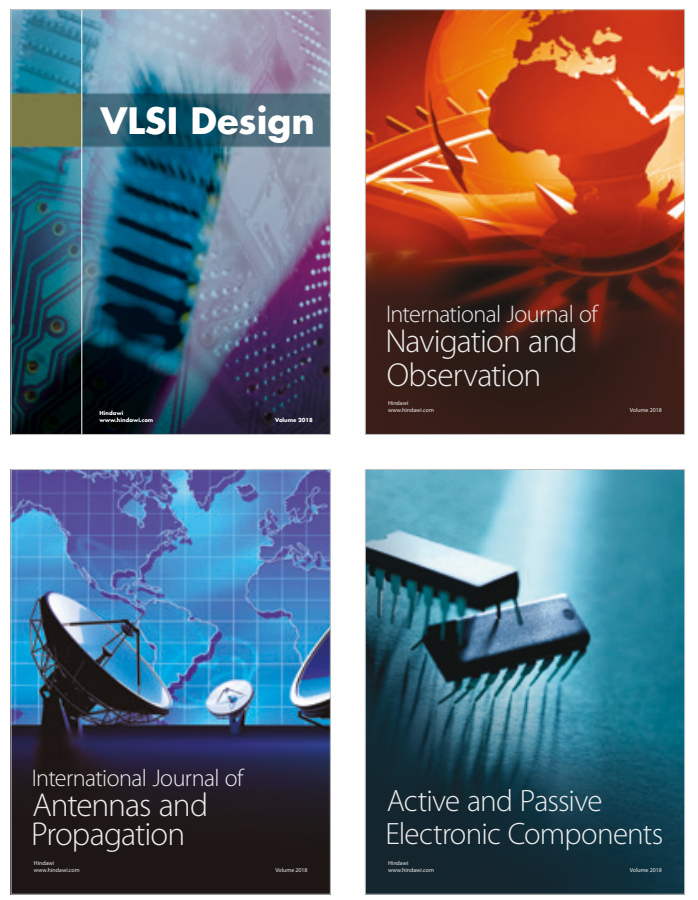
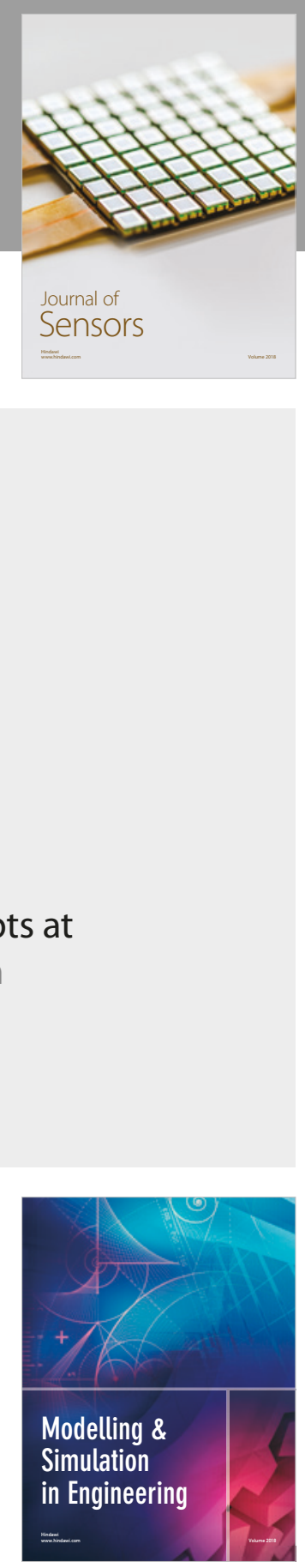

\section{Advances \\ Multimedia}
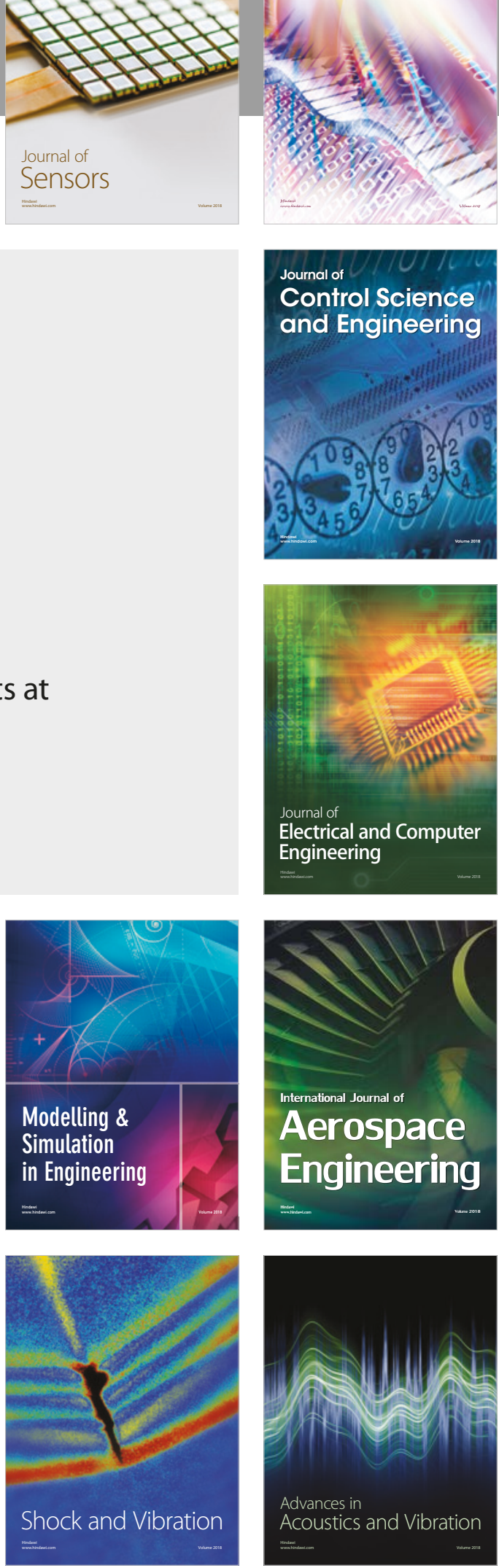